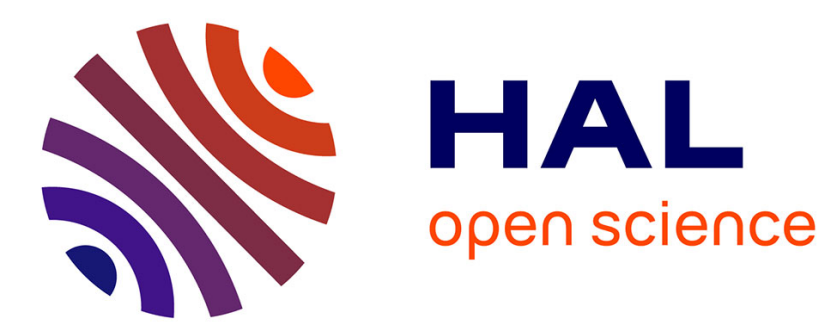

\title{
Devenirs identitaires dans les confins Sino-Tibétains : contextes et transformations \\ Stéphane Gros
}

\section{To cite this version:}

Stéphane Gros. Devenirs identitaires dans les confins Sino-Tibétains : contextes et transformations . Cahiers d'Extrême-Asie, 2014, Des mondes en devenir. Interethnicité et production de la différence en Chine du Sud-Ouest, 23, pp.63-102. hal-01391804

\section{HAL Id: hal-01391804 https://hal.science/hal-01391804}

Submitted on 3 Nov 2016

HAL is a multi-disciplinary open access archive for the deposit and dissemination of scientific research documents, whether they are published or not. The documents may come from teaching and research institutions in France or abroad, or from public or private research centers.
L'archive ouverte pluridisciplinaire HAL, est destinée au dépôt et à la diffusion de documents scientifiques de niveau recherche, publiés ou non, émanant des établissements d'enseignement et de recherche français ou étrangers, des laboratoires publics ou privés. 


\title{
DEVENIRS IDENTITAIRES DANS LES CONFINS SINO-TIBÉTAINS : CONTEXTES ET TRANSFORMATIONS
}

\author{
Stéphane GROs*
}

\begin{abstract}
This article discusses the fluidity of ethnic categorization in the context of changing ethnic relations, based on a diachronic and regional approach. It is argued that such an approach is needed to understand identity trajectories of peoples of the Sino-Tibetan borderlands. While studies on minorities in China are often limited to one ethnic group, the perspective defended here considers the interactions between several groups in specific contexts, and at different scales, which contribute to determine shifts in identity. The analysis focuses on the Sino-Tibetan borderlands of today's Sichuan and Yunnan provinces, and the diverse and often Tibetanized groups located in these mountains. Taking into account the changing socio-political environment, mainly from the late Qing to the People's Republic of China, belps us to uncover a number of factors determining the identity formation of the groups under consideration, and the persistence of a form of genealogical thinking. A range of cases is considered to provide comparative data at the regional level, supporting the degree of generality of the lability of ethnic categorization as well as identity changes, and the disjunctions between assigned categories, culture, language, and local identity.
\end{abstract}

\section{Introduction}

Cet article traite de l'ethnonymie régionale et de son évolution concernant un ensemble de peuples situés le long desdites marches sino-tibétaines, au carrefour des actuelles provinces du Sichuan, du Yunnan et de la région autonome du Tibet. Alors que les études sur les minorités en Chine sont souvent limitées à un groupe ethnique unique, il semble nécessaire de prendre en compte les interactions entre plusieurs groupes dans des contextes spécifiques, et à différentes échelles, pour être à même de déterminer les changements d'identité et de catégorisation dans leurs relations à l'environnement socio-politique. Couvrant principalement la période allant de la fin des Qing 清 (I644-I9II) à la République populaire de Chine, je concentre mon analyse sur la mixité constitutive de cette région sino-tibétaine et identifie un certain nombre de facteurs qui déterminent les transformations identitaires des

* Je tiens à remercier Sylvie Beaud, Katia Buffetrille, Katia Chirkova, Guillaume Jacques, Edwin Schmitt, et les deux évaluateurs anonymes dont les suggestions m'ont été très profitables. Cet article a été écrit dans le cadre du projet « Territories, Communities, and Exchanges in the Kham Sino-Tibetan Borderlands » (Starting Grant n 283 870), financé par le Conseil européen de la recherche (ERC).

Cabiers d'Extrême-Asie 23 (2014) : 63-102

(c) École française d'Extrême-Orient, Paris, 2015 Do not circulate without permission of the editor / Ne pas diffuser sans autorisation de l'éditeur 
divers groupes souvent tibétanisés qui habitent ces montagnes. La finalité d'une telle approche est d'étudier non pas la distribution de traits culturels, mais la matrice sociale et historique et les « chaînes de sociétés » qui sont le creuset de l'ethnicitér.

Voilà bientôt un demi-siècle, Rolf Stein (I9II-1999) publiait deux études importantes consacrées aux confins entre Chine et Tibet $^{2}$. Dans Les tribus anciennes des marches sino-tibétaines, il faisait le point sur les dénominations, classifications et légendes d'un ensemble de «tribus » dispersées le long de la bordure orientale du haut plateau en mettant à profit les sources tibétaines restées à ce sujet peu exploitées à l'époque. Stein avait identifié dans les textes tibétains la tradition historiographique relative à « six tribus primitives » (proto-clans) qu'il tentait de replacer dans l'histoire et de localiser, essentiellement au Nord-Est du Tibet. Le présent article tire son inspiration de l'une des conclusions de Stein, par laquelle il affirmait que la tradition tibétaine attribue une prépondérance aux tribus des marches sino-tibétaines, dont les migrations « ont grandement contribué à la formation du complexe ethnique et culturel de ce qu'on entend par le Tibet à l'époque historique $»^{3}$. La référence à ce « complexe ethnique et culturel » tibétain souligne avec justesse l'existence d'une diversité interne, très significative tout au long de la bordure orientale du plateau tibétain qui constitua aussi une interface où d'autres ethnicités ont émergé et se sont développées au fil des siècles.

Les fluctuations de nomenclature relevées par Stein sont parallèles à celles des termes chinois appliqués aux peuples des mêmes régions. Dans les deux cas, l'identification précise de ces peuples reste une gageure, et la complexité de leurs relations et de leurs migrations à travers l'histoire continue d'être l'objet de plus de questions que de réponses. L'évolution de l'ethnonymie est à la mesure de cette complexité ethnique, culturelle et linguistique des marches sino-tibétaines.

Cette vision de la bordure sino-tibétaine comme creuset ethnique et culturel trouve écho dans des recherches menées plus récemment en Chine. Sous l'impulsion de Fei Xiaotong 費孝通 (1910-2005) s'est développé un vif intérêt pour cette région où il entrevoyait la possibilité de saisir les liens historiques comme linguistiques qui unissent les groupes en présence, les traces d'un continuum socioculturel entre les divers peuples de langues tibéto-birmanes, depuis les Qiang de la bordure nord-est du plateau tibétain jusqu'aux Jingpo (Jinghpaw) de la frontière sino-birmane au sud par exemplet. Cette région, Fei Xiaotong la désignait par l'expression de « couloir tibéto-yi » (Zang-Yi zoulang 藏彝走廊) : bordé à l'ouest par l'aire de culture tibétaine et à l'est par l'aire de culture yi (et chinoise han), ce couloir s'étend de l'actuelle

I. J'emprunte l'expression de «chaînes de sociétés » à Jean-Loup Amselle, Logiques métisses. Anthropologie de l'identité en Afrique et ailleurs, Paris, Payot, 1990.

2. Rolf A. Stein, «Les K'iang des marches sino-tibétaines, exemple de continuité de la tradition ", dans École pratique des hautes études, Section des sciences religieuses, Annuaire 19571958, Paris, Imprimerie nationale, 1956, p. 3-15 ; Les Tribus anciennes des marches sino-tibétaines, Université de Paris, Faculté des Lettres, Imprimerie Nationale, 1959.

3. R. Stein, Les tribus anciennes, p. 84-85.

4. Voir Fei Xiaotong, 《Guanyu woguo minzu de shibie wenti » 䏌于我国民族的识别问题 (Ethnic Identification in China), Zhongguo shebui kexue 中国社会科学 (Social Science in China), n I I (1980), p. I47-I62.

(C) École française d'Extrême-Orient, Paris, 2015

Do not circulate without permission of the editor / Ne pas diffuser sans autorisation de l'éditeur 
province du Gansu au nord jusqu'au nord-est de l'Inde au sud, englobant le nord du Yunnan et de la Birmanies.

L'idée d'un continuum repose en partie sur le présupposé que l'un des mouvements migratoires de ces groupes tibéto-birmans, selon le critère linguistique, prit sa source à l'est du plateau tibétain pour essaimer progressivement vers le sud le long de la bordure sichuanaise, des montagnes du Yunnan et du nord de la Birmanie, et jusqu'à l'est de la chaîne himalayenne. C'est en somme une version plus englobante que la tradition tibétaine évoquée par Stein, qui associe aux Tibétains un ensemble d'autres peuples qui partageraient une origine commune. Nombre d'historiens ou d'ethnologues tracent ainsi de manière conventionnelle l'origine de bien des peuples de langues tibéto-birmanes localisés aujourd'hui au Sichuan ou au Yunnan depuis le nord-est du plateau tibétain, et considèrent comme leurs ancêtres les groupes qui y nomadisaient il y a de cela près de deux millénaires. Très discutable, cette théorie devenue orthodoxie s'inscrit dans une pensée généalogique — présente dans le discours académique comme dans les représentations locales - qui peine à prendre en compte la mixité et les transformations identitaires que le présent article cherche à mettre au jour.

La perspective adoptée ici vise à replacer cette zone de contact comme une double périphérie, de la Chine et du Tibet considérés comme centres de civilisation. En tant que tels, ils avaient chacun leur vision d'un monde allogène barbare et c'est à la fois les processus de sinisation et de tibétanisation qu'il est important de prendre en compte, au-delà de leurs différences, pour pouvoir comprendre la manière dont des identités régionales, linguistiques et ethniques, se sont forgées et ont évolué jusqu'à l'époque contemporaine. Or, si les études portant sur l'ethnicité en Chine ont connu un essor certain depuis les années I990, cela contraste fortement avec le peu d'attention accordé à cette question dans le champ des études tibétaines ${ }^{6}$. Il semble pourtant nécessaire de réfléchir à la diversité interne de ceux désignés comme « Tibétains » aujourd'hui en Chine $\mathrm{C}^{7}$ ce à quoi une plongée au cœur des marches sino-tibétaines peut contribuer.

Historiquement, les identités de groupe se forment et se transforment sous l'effet des migrations, du commerce, de la conquête ou de la colonisation, etc., dans un contexte relationnel et changeant. Il est aujourd'hui impossible en République populaire de Chine de faire abstraction du discours officiel de l'État-Parti concernant

5. L'idée de ce « couloir» (que l'on retrouve traduit en anglais par « ethnic corridor») a largement été reprise par divers historiens et linguistes chinois ; voir la discussion dans l'introduction à ce volume, et infra.

6. L'une des contributions récentes à cette question de la prise en compte du rapport entre centre et périphérie au Tibet est l'ouvrage édité par P. Christiaan Klieger, éd., Tibetan Borderlands. Proceedings of the Tenth Seminar of the IATS, 2003, vol. 2, Leiden, Brill, 2006.

7. Car si cette catégorie, dans le contexte de la politique des nationalités en Chine, se voit assigner un marqueur " minoritaire ", dans le contexte du discours nationaliste en revanche, comme S. Shneiderman l'a justement souligné, elle acquiert "l'invisibilité de la dominance " comme majorité en soi. Voir S. Shneiderman, "Barbarians at the Border and Civilising Projects: Analysing Ethnic and National Identities in the Tibetan Context," dans P. C. Klieger, éd., Tibetan Borderlands, Proceedings of the Tenth Seminar of the IATS, 2003, p. 9-34. 
les 55 《 nationalités minoritaires》 (shaoshu minzu 少数民族) au rang desquelles figurent les Tibétains (Zangzu 藏族) ${ }^{8}$. La présence de l’État moderne vient fortement influer sur la manière spécifique dont les référents identitaires peuvent être mobilisés, et contribue à redéfinir les identités. La langue, la culture et la parenté servent généralement de base à la différentiation, mais s'y ajoute aussi l'histoire - et sa juste interprétation. Les tensions entre les perceptions identitaires locales et l'attribution identitaire en fonction de la classification officielle des minorités chinoises en catégories de minzu (nationalités) est un sujet relativement bien documenté

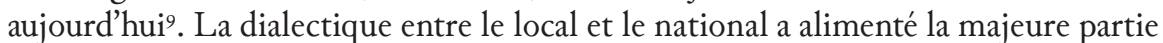
des contributions aux études sur l'ethnicité dans le Sud-Ouest de la Chine. Dans ce cadre, Stevan Harrell a cependant su rappeler l'importance de considérer l'ethnicité localement, dans le sens où les revendications identitaires sont en général formulées dans le contexte d'interactions au sein d'une communauté locale ${ }^{\text {Io }}$.

À partir de sources écrites anciennes ou contemporaines, chinoises et occidentales, je vais m'efforcer de dresser un tableau de la complexité taxinomique relative à certains de ces peuples desdites marches sino-tibétaines, complexité qui repose sur diverses conceptions quant aux critères servant à l'identification. Ce parcours de l'ethnonymie fera inévitablement référence aux catégories linguistiques et à leur influence, en soulignant que les frontières linguistiques ne sont pas forcément congruentes avec les frontières ethniques, et que l'émergence et l'affirmation des catégories ethniques - et la validation des catégories de minzu en l'occurrence est un processus complexe multifactoriel et souvent contingent. Un tel survol fait courir le risque de la généralisation superficielle, mais les cas évoqués sont suffisamment bien documentés pour servir l'entreprise comparative et s'autoriser une saisie à l'échelle régionale. Dans une première partie, l'approche de l'ethnonymie permet ainsi de souligner que les formulations identitaires locales et les catégorisations

8. Pour une discussion de la notion de minzu 民族, parfois aussi compris comme équivalent de "groupe ethnique », voir l'introduction à ce numéro. Sur son ambiguïté, qui recouvre à la fois les concepts de nation et de race, voir Frank Dikötter, The Discourse of Race in Modern China, Stanford, Stanford University Press, 1992. Voir également Joël Thoraval, «Le concept chinois de nation est-il 'obscur' ? À propos du débat sur la notion de minzu dans les années I980 ", Bulletin de sinologie, vol. 65, 1990, p. 24-4I.

9. Les recherches sur l'ethnicité en Chine on notamment été marquées par la publication des études comme celles de Melissa J. Brown, éd., Negotiating Ethnicities in China and Taiwan, Berkeley, University of California Press, 1996 ; Stevan Harrell, "Ethnicity, Local Interests, and the State: Yi Communities in Southwest China," Comparative Studies in Society and History, vol. 32, no 3 (1990), p. 515-548 ; S. Harrell, éd., Cultural Encounters on China's Ethnic Frontiers, Seattle, University of Washington Press, 1995 ; Katherine P. Kaup, Creating the Zhuang. Ethnic Politics in China, Boulder, Lynne Rienner Publishers, 200o. Pour des études de cas relatifs à la région sino-tibétaine, voir par exemple, S. Harrell, Ways of Being Ethnic in Southwest China, Seattle, University of Washington Press, 200I ; Koen Wellens, Religious Revival in the Tibetan Borderlands: The Premi of Southwest China, Seattle, University of Washington Press, 20Io ; Katia Chirkova, "Between Tibetan and Chinese: Identity and Language in the Chinese Southwest," South Asia: Journal of South Asian Studies, vol. 30, n 3, 2007, p. 405-417.

Io. Voir S. Harrell, "Ethnicity, Local Interests, and the State" ; et plus particulièrement, Ways of Being Ethnic.

(C) École française d'Extrême-Orient, Paris, 2015

Do not circulate without permission of the editor / Ne pas diffuser sans autorisation de l'éditeur 
établies par les discours savants partagent une mentalité généalogique. Je m’attache à déconstruire, à travers une critique de ce que je nomme l'histoire régressive, l'idée que les groupes ethniques d'aujourd'hui sont les descendants directs de peuples historiquement identifiables. L'inscription de ces peuples dans l'histoire, les discours exogènes et endogènes relatifs à l'origine et aux migrations serviront à illustrer et discuter des devenirs identitaires des groupes pris en considération, de leur ethnogénèse comme de leur disparition dans la taxinomie. La seconde partie de cet article s'attachera alors à explorer certains de ces destins taxinomiques, et la manière dont la question des origines, des différences culturelles comme linguistiques, influent sur les processus de choix ou d'assignation identitaire.

\section{Ethnonymie et généalogie : des barbares aux shaoshu minzu}

Comme bien d'autres civilisations, la Chine et le Tibet, à travers leur histoire, se sont considérés comme des centres entourés de peuples allogènes barbares. Les visions et représentations de l'Autre, distinct mais assimilable, et les types de relations associées, constituent ce que Magnus Fiskesjö a justement appelé une xénologie" Sans pouvoir faire ici l'étude des soubassements idéologiques de cette xénologie, il peut être utile de rappeler que dans les sources chinoises, plusieurs dénominations génériques et souvent interchangeables étaient utilisées pour désigner les barbares. Ainsi dans le Liji, le Livre des rites ( $\mathrm{v}^{\mathrm{e}}$ av. J.-C.), les Zhou sont situés au centre et les barbares sont associés aux quatre directions cardinales : Yi de l'Est, Di du Nord, Rong de l'Ouest, et Man du Sud (Dongyi 东夷, Beidi 北狄, Xirong 西戎, Nanman 南蠻). Après la fondation de l'empire des Qin 秦 (22I-206 av. J.-C.), et sous les Han 漢 (206 av. J.-C.-220 ap. J.-C.), ces termes génériques restent globalement les mêmes, avec cependant une grande variabilité des usages et combinaisons des termes, souvent associés par paires et parfois organisés en séries numérales ${ }^{12}$. La pérennité de certaines appellations masque donc la grande variabilité de leur référent, ce qui vaut également comme nous le verrons pour les terminologies des époques plus récentes.

La dynamique de transformation (bua 化) par la civilisation émanant du centre comme une série de cercles concentriques jusqu’à sa périphérie (buawai 化外), où au centre civilisé succède la sphère des barbares « cuits ", shu 熟 (civilisés), puis celle des barbares «crus», sheng 生 (sauvages), s'applique particulièrement auxdits Barbares du Sud, Nanman 南蠻. La tradition impériale favorisait la reconnaissance explicite des groupes allogènes qui représentaient une menace justifiant l'imposition

II. Xenos en grec peut désigner à la fois l'étranger et l'hôte, et dénoter une relation réciproque, à la base du concept d'hospitalité. Cf. Magnus Fiskesjö, "On the 'raw' and the 'cooked' barbarians of imperial China," Inner Asia, n ${ }^{\text {os }} \mathrm{I}-2$, I999, p. I39-168.

I2. Voir par exemple Zhitang Drocourt, «Des simples sauvages aux redoutables étrangers : la notion de «barbares » en Chine ancienne à travers leurs dénominations », dans Isabelle Rabut, éd., Visions du «barbare » en Chine, en Corée et au Japon, Actes de la journée d'étude organisée le 3I mars 2008 par le Centre d'Études Chinoises et le Centre d'Études Japonaises de l'INALCO, Paris, INALCO Éditions, 2010, p. 13-28. 
du pouvoir impérial, et constituaient les sujets principaux de la bienveillance civilisatrice ${ }^{\mathrm{I}}$. La diffusion d'un modèle culturel et rituel, la mise en ouvre de diverses formes de standardisation au fil des dynasties successives, contribuèrent à l'unité politique de la Chine ${ }^{14}$.

L'empire tibétain formulait également sa version d'une supériorité culturelle et associait des stéréotypes dépréciatifs à certains ethnonymes désignant des groupes considérés comme barbares. Le terme Mon ou Mon-pa, dont le chinois Man pourrait être à l'origine, était appliqué aux populations des régions au-delà de la sphère d'influence culturelle et religieuse, notamment au sud sur les versants boisés de l'Himalaya. D'autres populations tribales méridionales furent aussi appelées Lopa (tib. lHo-ba, « Méridionaux », ou Klo-pa, « Barbares ») ou encore Lalo (tib. Kla-klo) ${ }^{15}$. Termes génériques plutôt que noms de peuples, ils témoignent de ce que Françoise Pommaret a décrit comme « une attitude condescendante et méprisante » vis-à-vis de ceux associés à l'image de non-bouddhistes, sans véritable « culture $»^{16}$.

Dans le cas de la Chine comme du Tibet, l'existence de "projets civilisateurs ", pour différents qu'ils soient, conditionnent les visions d'un monde allogène aux limites de la civilisation ${ }^{17}$. Les régions à l'est du plateau, bien qu'également périphériques, ont cependant une place particulière dans la tradition tibétaine. En effet, les semi-mythiques « tribus » des marches sino-tibétaines sur lesquelles R. Stein s'était penché en vue de les relier à des peuples historiquement connus, seraient originaires du Tibet oriental. Selon la tradition séculaire présentée comme une généalogie, ces lignages ancestraux sont généralement regroupés par six, ou quatre ${ }^{18}$. Issus d'ancêtres

I3. Une discussion de la notion de civilisation (wenming 文明) dépasse largement l'objet de cet article. Le lecteur pourra cependant bénéficier de la présentation par Tang Yun et Zhang Yuan dans ce numéro de l'ouvrage de Wang Mingming 王铭铭, Zhong jian quan: «Zang-Yi zou lang » yu ren lei xue de zai gou si 中间圈—— “藏彝走廊” 与人类学的再构思 (The intermediaries: Tibetan-Yi corridor and the reformation of anthropology), Beijing, Shehui kexue wenxian chubanshe, 2008.

I4. Sujet bien vaste et souvent renouvelé au sein des études chinoises; voir par exemple les débats suscités autour des notions d'orthopraxie et d'hétéropraxie, revisitées dans le numéro spécial de Modern China (2007) : Donald Sutton, "Ritual, Cultural Standardization, and Orthopraxy in China: Reconsidering James L. Watson's Ideas,” Modern China, vol. 33, nº 3, 2007, p. 3-2I.

15. Terme dérivé du sanscrit mleccha. Au sujet de ces appellations tibétaines, voir notamment Françoise Pommaret, "The Mon-pa revisited: In search of Mon," dans Toni Huber, éd., Sacred Spaces and Powerful Places in Tibetan Culture. A Collection of Essays, Dharamsala, Library of Tibetan Works and Archives, 1999, p. 52-73. Le Klo-pa tibétain est à l'origine de l'actuel Luoba 珞巴 des Chinois, exonyme devenu nom officiel désormais attribué à un groupe reconnu comme l'une des nationalités minoritaires de la République Populaire de Chine. On trouve aussi en tibétain l'usage du terme de Hlo ka tra (tib. 1Ho-kha-khra, ou Klo-kha-khra), « Méridionaux au visage (à la bouche) strié(e)».

I6. F. Pommaret, "The Mon-pa revisited," p. 53.

17. J'emprunte la notion de "projet civilisateur » à S. Harrell, qu'il définit comme une forme d'interaction entre un centre civilisateur et des peuples périphériques reposant sur une inégalité. Voir S. Harrell, "Introduction: Civilizing Projects and the Reaction to Them," dans S. Harrell, éd., Cultural Encounters on China's Etbnic Frontiers, p. 4.

I8. Soit I. dBra ; 2. IDong ; 3. 'Bru ; 4. 1Ga ; 5. dPa'; 6. mDa'. Ou pour la série de quatre : I. Se ; 2. IDong; 3. sTong; 4. dMu. Je ne restitue pas ici les différentes orthographes sous lesquelles 
divins, ils seraient à l'origine du peuple tibétain, et auraient ainsi essaimé à travers le Tibet à partir des régions du Kham et de ses environs. Si bien que selon R. Stein, « [1] es Chinois n'avaient donc pas tort de rattacher les Tibétains T'ou-fan [Tufan] du Centre aux K'iang [Qiang] de l'Est. Les deux groupes ne sont pas identiques, mais le dernier a été un des principaux éléments qui ont contribué à la formation du premier. [...] La tradition tibétaine se justifie aussi bien dans les massifs boisés de l'Est tibétain et des marches chinoises. $»^{19}$

Le Qiang 姜 auquel Stein se référait, correspond à l'une de ces catégories génériques qui, comme celle de Man 蠻, ont perduré à travers les siècles. Sous les Han, Qiang désigne des populations qui occupent les régions à l'ouest de la Plaine centrale. Si Di 氏 et Qiang en vinrent à être étroitement associés pour désigner des peuples le long de la bordure nord-est du plateau tibétain de manière englobante, Wang Mingke a désormais démontré que Qiang était davantage un terme générique définissant des frontières culturelles et politiques avec les populations marginalisées vivant à l'ouest des Han (Huaxia 華夏) ${ }^{20}$. Qiang est longtemps resté une identité flottante dont l'usage suivit l'expansion des frontières de la culture Han, un terme utilisé depuis l'Antiquité pour se référer aux « autres » situés au-delà de la frontière occidentale.

L'association ultérieure courante des Di et des Qiang dans l'historiographie chinoise ne repose sur aucune évidence eu égard à une communauté de langue ou de formation politique. Bien que l'« ensemble » Di-Qiang ne soit qu'une construction sociale et politique née du processus d'interaction historique entre le centre (Hua) et sa marge, il est souvent considéré aujourd'hui comme la source ancestrale des peuples de langues tibéto-birmane que l'on retrouve le long de la bordure sinotibétaine jusqu'au Yunnan, comme nous le verrons ci-dessous. Le terme Qiang est depuis devenu le nom officiel de «nationalité minoritaire » d'un peuple des marches tibétaines du Sichuan. Si Qiang fut ainsi au fil des siècles un label régulièrement réassigné aux «barbares de l'Ouest » en fonction des fluctuations de l'expansion du centre, l'usage des termes Di, Rong, Man ou Yi, fut bien plus variable que la classification cardinale du Liji ne le suggérait.

ces noms apparaissent dans les sources. Voir R. Stein, Les tribus anciennes... ; ainsi que Tashi Gyatso Gyilung et Thugchock Dorji Gyilung, The Treasure of the Ancestral Clans of Tibet, traduit par Yeshi Dhondup, Dharamsala, Library of Tibetan Works and Archives, 2009.

19. R. Stein, Les Tribus des marches, p. 84.

20. Voir plus généralement Wang Mingke 王明珂, Hua-Xia bianyuan: Lishi jizi yu zuqun rentong 華夏邊緣——歷史記憶與族群認同 (Aux frontières de la Chine : Mémoire historique et identité ethnique), Beijing, Shehui kexue wenxian chubanshe, 2006 [1997] ; ainsi que Wang Mingke, Qiang zai Han-Zang zhi jian: Chuanxi Qiangzu de lishi renleixue yanjiu 姜在漢藏之間——西 芫族的歷史人類學研究 (Les Qiang entre Han et Tibétains : Une anthropologie historique des Qiang du Sichuan de l'Ouest), Beijing, Zhonghua Shuju, 2008 [2003], et Wang Mingke, "From the Qiang Barbarians to the Qiang Nationality: The Making of a New Chinese Boundary," dans Shu-min Huang et Cheng-kuang Hsu, éds., Imagining China: Regional Division and National Unity, Taipei, Institute of Ethnology, 1999, p. 43-80. Voir aussi la discussion des catégories Qiang et Di dans Terry F. Kleeman, Great Perfection: Religion and Ethnicity in a Chinese Millennial Kingdom, Honolulu, University of Hawai'i Press, 1998, p. 54-60.

(C) École française d'Extrême-Orient, Paris, 20I5 Do not circulate without permission of the editor / Ne pas diffuser sans autorisation de l'éditeur 
Alors que la connaissance des régions du sud-ouest progresse au fur et à mesure de l'expansion impériale chinoise, l'ethnonymie s'enrichit et se complexifie, mais reste généralement une exonymie. L'un des plus anciens documents historiques chinois qui se réfère spécifiquement à des groupes indigènes du sud-ouest (Xi'nan Yi 西南夷) est un chapitre des Annales historique (Shiji 史記) de Sima Qian 司馬遷 (I45-86 av. J.-C.). Intitulé «Xi'nan Yi liezhuan » 西南夷列傳, il y est fait référence à de nombreuses chefferies disséminées dans le Sud-Ouest, dont des dizaines de groupes disséminés le long de la bordure du plateau tibétain, tels que les Baima 白馬, faisant partie des Di 氏 ${ }^{21}$.

Plus tardif, le bien connu Livre des Man (Man sbu 蠻書), écrit par l'historien Fan Chuo 桥綽 sous les Tang (vers 860-873), traite de l'ensemble des dénominations et descriptions des peuples méridionaux ${ }^{22}$. La bordure sino-tibétaine se situait alors à l'interface entre trois grandes puissances : l'Empire chinois, l'Empire tibétain, et le royaume de Nanzhao (738-902). Certains passages du Man shu font état des différents peuples ou tribus qui se trouvaient alors dans la sphère du puissant royaume de Nanzhao qui occupait les rives du lac Erhai au nord-ouest du Yunnan. Au sein de l'ensemble des Nanman 南蠻, le Man shu mentionne notamment les Baiman 白蠻 et les Wuman 烏蠻, ces derniers étant généralement associés aux principautés (zhao 詔) qui composèrent le Nanzhao (liu zhao Wuman 六詔烏蠻, Wuman des six principautés) ${ }^{23}$.

2I. Cette dénomination de Baima se retrouve à l'heure actuelle, comme nous le discuterons plus loin dans cet article. Un nombre important de documents historiques pourraient être cités, mais je ne ferai ici référence qu'à quelques sources les plus significatives. Concernant les textes relatifs aux Xi'nan Yi, Yang Chengzhi 杨成志 donnait une liste non-exhaustive de 47 sources historiques (allant de la dynastie des Han à la période républicaine) ; voir Yang Chengzhi 杨成志, «Xi'nan minzu de yanjiu » 西南民族的研究 (Étude sur les groupes ethniques du sud-ouest de la Chine), dans Liu Shaorui 刘邵瑞, éd., Yang Chengzhi wenji 杨成志文集, Guangzhou, Zhongshan daxue chubanshe, 2004 [1932], p. 132-I33. Tao Yunkui 陶云迬 listait pour sa part 35 sources historiques (des Han aux Qing), mais passait également en revue de manière détaillée les écrits de la période républicaine, y compris les nombreuses sources occidentales ; voir Tao Yunkui 陶云迬, "Yunnan tuzhu minzu yanjiu zhi huigu yu qianzhuan » 雲南土著民族研究之回顧與前瞻 (Rétrospective et perspectives des études sur les ethnies indigènes du Yunnan), dans Tao Yunkui, Tao Yunkui minzu yanjiu wenji 陶云迬民族研究文集, Beijing, Minzu chubanshe, 2012 [194I], p. 92-IIO.

22. Fan Chuo, Man shu, traduit en anglais par G. H. Luce sous le titre Man sbu, Book of the Southern Barbarians, [G.P. Oey, éd.] Southeast Asia Program, Department of Far Eastern Studies, Cornell University, Ithaca, New York, I96I.

23. À l'Ouest, se trouvaient les Xunchuan 尋傳 (" [Ceux qui] cherchent [des terres ?] »), Luoxingman 裸形蠻 《Barbares nus » et les Yeman 野蠻 《Barbares sauvages »; plus au nord se trouvaient les Shunman 順蠻, les Shiman 施蠻, les Heman 和蠻 et les Mosoman 磨些蠻 qui fondèrent des principautés unifiées au sein du Nanzhao. Cf. You Zhong 尤中, Yunnan minzu shi 雲南 民族史, Kunming, Yunnan daxue chubanshe, I994, p. II3-II7 ; Wilfrid Stott, "The expansion of Nan-chao Kingdom," T'oung Pao, vol. L, nos. I-3, 1963, p. 190-220. Le nom Mo-so (ou encore Mosuo en chinois) est toujours en usage aujourd'hui ; il était orthographié de différentes manières selon les sources, et on sait que le caractère xie 些 doit dans ce cas se lire so [suo] ; cf. Paul Pelliot, « Deux itinéraires de Chine en Inde à la fin du viII e siècle », Bulletin de l'École française d'Extrême-Orient, vol. 4, n ${ }^{\text {os }} \mathrm{I}-3$, I904, p. I70, n. 9 ; Edouard Chavannes, "Documents historiques et géographiques relatifs à Li-Kiang,” dans Jacques Bacot, Les Mo-So, Leiden, E. J. Brill, I9I3, 
Il est courant de retrouver dans la classification des peuples non-han du sud de la Chine un code de couleurs relatif soit à un statut ou une strate sociale, soit simplement aux caractéristiques vestimentaires. La catégorisation en blanc et noir existe par ailleurs dans les catégories indigènes. Ainsi le qualificatif de «noir» constitue la racine de certains ethnonymes des peuples de ces régions, tel naq (Naxi 納西), ou nuo (Nuosu, Yi 彝), ou au contraire «blanc » comme dans pre (Premi, Pumi 普米), ou les actuels Baizu 白族 de la région de Dali 大理 (cependant historiquement connus sous le nom de Minjia 民家).

Certains ethnonymes furent utilisés dans les sources chinoises en référence à des ensembles géographiquement et parfois culturellement cohérents, tel celui de Xifan 西蕃. Ce dernier nous intéresse plus particulièrement, en tant que terme spécifique appliqué aux peuples de la bordure sino-tibétaine. Si le terme Fan 蕃 (ou 番) est très ancien et désignait plus généralement certains « barbares » ou « étrangers », pour ce qui nous concerne son usage devint plus courant sous les Tang (6I8-906) en relation au terme Tufan 吐蕃 (ou Tubo) alors couramment utilisé pour désigner les Tibétains ${ }^{24}$. À cette époque Xifan en vient à être appliqué à un ensemble de populations de la bordure sino-tibétaine, de culture et de religion proche des tibétains, mais parlant des langues différentes. Le terme Xifan resta d'usage courant jusqu'à l'époque moderne, et servit à désigner un sous-groupe dans les classifications linguistiques. $\mathrm{Au}$ Yunnan, des groupes tibétophones étaient aussi identifiés, et ce jusqu'au début des années cinquante, sous le nom de Guzong 古宗 (估倧) qui a depuis disparu de l'ethnonymie régionale, mais dont l'usage était proche de la catégorie Xifan ${ }^{25}$.

De cette ethnonymie approximative, on peut retenir que les marges sinotibétaines situées au Sichuan ou au Yunnan sont longtemps restées méconnues malgré leur position à l'interface des mondes chinois et tibétain. La topographie n'y est pas indifférente, justifiant l'adage «les montagnes sont hautes, l'Empereur est loin » (shan gao buangdi yuan 山高皇帝遠). La prise en main administrative plus directe de cette région frontière sous les Qing aboutit à une cartographie plus précise de la diversité des peuples habitant ces régions, laquelle se précisa au cours de la période

p. I32; Han-Fa zonghe cidian 漢法綜合詞典 (Dictionnaire français de la langue chinoise), Institut Ricci, Kuangchi Press, 1986, caract. I9I5 [b].

24. À propos de l'appellation Tufan, voir par exemple R. Stein, La Civilisation tibétaine, Paris, L'Asiathèque, 1987, p. 9. Tufan (ou Tubo) serait une transcription chinoise d'un terme turc pour le Tibet *Töpän/*Töpüt, signifiant « sommet, hauteur, hautes terres »; voir Louis Bazin et James R. Hamilton, "L'origine du nom Tibet », dans Ernst Steinkellner, éd., Tibetan History and Language: Studies Dedicated to Uray Géza on His Seventieth Birthday, Vienne, Arbeitskreis für Tibetische und Buddhistische Studien, Universität Wien, I99I, p. II-I7. Pour une intéressante discussion des termes Fan, Tufan et Han, voir Yang Shao-yun, 20I4, "Fan and Han: The Origins and Uses of a Conceptual Dichotomy in Mid-Imperial China, ca. 500-I200," dans Francesca Fiaschetti et Julia Schneider, éds., Political Strategies of Identity-building in Non-Han Empires in China, Wiesbaden, Harrasowitz Verlag, p. 9-36.

25. Xifan en vint à désigner de manière conventionnelle un ensemble de groupes que l'on pourrait aujourd'hui rapprocher du groupe linguistique Qiangique encore mal identifié ; voir infra. Les Guzong, souvent assimilés au actuels Pumi, étaient aussi distingués par les appellations de chou Guzong 臭古宗 (assimilés aux Moso Guzong) et de xiao Guzong 小古宗 (les petits Guzong). 
républicaine. Si des nomenclatures déjà complexes existaient depuis des temps anciens - dont je n'ai pu rendre pleinement compte dans ce rapide survol - des classifications nouvelles virent le jour qui, plus qu'elles ne renseignent sur la diversité ethnique de ces régions, nous indiquent l'attention croissante dont elles furent l'objet pour des raisons essentiellement de contrôle politique.

\section{Les sciences sociales face à la diversité ethnique}

La période moderne puis contemporaine, après l'avènement de la République populaire de Chine (1949), est la plus marquante concernant l'émergence d'une nouvelle taxinomie qui, par contraste avec les classifications essentiellement culturelles d'autrefois, établit les bases (scientifiques) des catégories de minzu. C'est alors, avec le développement des disciplines archéologiques, historiques et ethnologiques, de nouvelles perspectives et modes d'appréhension de la différence qui s'instaurent. Dans cette reconfiguration, il existe une continuité certaine entre la République (I9I2) et la République populaire (1949), où l'on retrouve une nécessité de nommer et de classer propre à une approche naturaliste caractéristique d'un contexte scientifique en pleine évolution.

Parmi ceux qui s'intéressèrent aux questions de dénomination et de classification des peuples des frontières, Yang Chengzhi 杨成志 (1902-199I), dans un article sur les études relatives aux ethnies du Sud-Ouest, avait établi une liste de noms recueillis dans les sources historiques, et en rassemblait I40 pour le seul Yunnan ${ }^{26}$. Yang Chengzhi soulignait à cette occasion le manque de rigueur scientifique des terminologies existantes en Chine, et exhortait ses collègues à s'inspirer des études occidentales comme des nombreux ouvrages écrits par les missionnaires, et donnait en exemple les divisions linguistiques établies à l'époque. L'historien Ma Changshou 馬長壽 (I906-I97I) avait également donné comme exemple pour la province du Guizhou une liste de 82 noms, qu'il qualifiait d'« énumération » non-organisée pour souligner le manque de principe directeur permettant la classification de ces peuples ${ }^{27}$.

Pendant la période républicaine, comme James Leibold l'a clairement montré, un ensemble d'ethnologues de la nouvelle génération souvent formés à l'étranger contribuèrent alors à revisiter la définition du concept de minzu donnée par Sun Yat-sen, en insistant sur la diversité interne de la Chine, ce dont les travaux de Gu Jiegang 顧頡岡 (1893-1980) sont particulièrement représentatifs ${ }^{28}$. Plusieurs, tels que Yang

26. Sur la base du Yunnan tongzhi gao 雲南通志搞, «Nanman zhi » 南蠻志 (juan 172 à 190), voir Yang Chengzhi, «Xi’nan minzu de yanjiu », p. I29-I30. Concernant Yang Chengzhi, l'un des premiers ethnologues de terrain en Chine, voir Gregory E. Guldin, The Saga of Anthropology in China: From Malinowski to Moscow to Mao, Londres, M. E. Sharpe, 1993, p. 50-55.

27. Noms figurant dans le Qian Miao tu shuo 黔苗圖說; voir Ma Changshou馬長壽, «Zhongguo Xi'nan minzu fenlei » 中國西南民族分類 (Classification des groupes ethniques du Sud-Ouest de la Chine), dans Ma Changshou, Ma Changshou minzu xue lunwen ji 馬長壽民族學論文集, Beijing, Renmin chubanshe, 2003 [1936], p. 54-55.

28. Concernant Gu Jiegang, voir plus particulièrement Hon Tze-Ki, "Ethnic and Cultural Pluralism: Gu Jiegang's Vision of a New China in His Studies of Ancient History," Modern China, vol. 22, n 3, 1996, p. 315-339 ; et James Leibold, Reconfiguring Chinese Nationalism: 
Chengzhi, soulignèrent l'importance des classifications linguistiques comme modèle taxinomique. À ce propos, Thomas Mullaney a montré que l'ouvrage en anglais sur le Yunnan de Henry Rodolph Davies, officier britannique, fut une source d'inspiration majeure pour les chercheurs chinois dans leurs tentatives de classification dès le début des années vingt ${ }^{29}$. Plus généralement, ces derniers se rendaient compte du travail ethnographique et archéologique accompli dans les régions frontières par les occidentaux. Ce qui faisait le plus défaut à l'époque était la connaissance fine des réalités du terrain, et des découpages identitaires locaux. Le missionnaire baptiste, ethnologue et archéologue, David Crockett Graham fut ainsi, selon les mots de Jeff Kyong-McClain et Geng Jing « un pionnier de la classification ethnique (minzu sbibie 民族識別) au Sichuan ", et avait acquis un statut certain dans les cercles académiques provinciaux où ses travaux faisaient référence ${ }^{30}$. Il exerça une influence notable dans le développement de la discipline ethnologique et des institutions qui se mettaient en place à l'époque au Sichuan. Au sein de ces institutions, Li Anzhai 李安宅 (1900-1985) joua un rôle de premier plan, notamment dans l'essor des études tibétaines ${ }^{31}$.

Très au fait de l'avantage acquis par les occidentaux, Ren Naiqiang 任乃強 (I894-1989) réalisa des enquêtes, au début des années 1930, qui contribuèrent significativement à améliorer les connaissances relatives aux régions où vivaient les Tibétains du Kham et d'autres groupes tels les Qiang au nord et les Naxi au sud ${ }^{32}$. Il est significatif que Ren Naiqiang, imprégné du contexte socio-politique de son époque, forgea l'appellation Xikang minzu 西康民族, qui associe au nouveau concept

How the Qing Frontier and its Indigenes Became Chinese, New York, Palgrave Macmillan, 2007, p. I22-I35.

29. Henry R. Davies, Yünnan: The Link Between India and the Yangtze, Cambridge, Cambridge University Press, 1909. La première référence importante à Davies dans l'ethnologie chinoise peut être trouvée dans les travaux de Ding Wenjiang 丁文江 (I887-1936), qui prononça une conférence universitaire sur les tribus du Yunnan dans laquelle sa taxinomie est presque entièrement dérivée du travail de l'officier britannique ; voir Thomas Mullaney, Coming to Terms with the Nation. Ethnic Classification in Modern China, Berkeley, University of California Press, 20II, p. 54. Ma Changshou dans son étude sur les classifications des ethnies du Sud-Ouest évoque les travaux des français comme des britanniques, et reproduit intégralement la classification linguistique donnée par H. Davies ; cf. Ma Changshou, «Zhongguo Xi'nan minzu fenlei », p. 59-6o.

30. Jeff Kyong-McClain et Geng Jing, "David Crockett Graham in Chinese Intellectual History: Foreigner as Nation Builder," dans D. M. Glover, S. Harrell, C. F. McKhann, et M. B. Swain, éd., Explorers and Scientists in China's borderlands 1880-1950, Seattle, University of Washington Press, 20II, p. 2II-239.

31. D. Crockett Graham joua ainsi un rôle important dans la formation de ce que d'aucuns appellent « l'École de Huaxi », d'après le nom de l'Université de Huaxi (et les institutions connexes, musée et centre de recherche sur les frontières) que des missionnaires américains, canadiens et britanniques contribuèrent à fonder dès I9IO ; voir Li Shaoming 李紹明, «Zhongguo renleixue de Huaxi xuepai »中國人類學的華西學派, Zhongguo renlei xue pinglun 中國人類學評論, $\mathrm{n}^{\circ} 4$, 2007, p. $4 \mathrm{I}^{-}-63$.

32. D'autres chercheurs chinois de l'époque, tels Ma Changshou 馬長壽, déjà mentionné, Xu Yitang 徐益棠 (I896-I953) et Lin Yaohua 林耀華 (I9OI-2000), furent parmi les premiers, dans les années 1930 et 1940, à réaliser des enquêtes de terrain et à contribuer au développement des connaissances sur les peuples de la région sino-tibétaine. 
de minzu le nom d'une non moins nouvelle (et éphémère) province chinoise, celle du Xikang ${ }^{33}$. Il précisait que cette catégorie englobait ceux appelés localement Kangfan 康番 (c'est-à-dire tibétains du Kham), mais que les immigrés Han appelaient plus communément Manzi 蠻子. Il établissait par ailleurs une claire différence entre les Tubote 土波特 (Tibétains) et les Xifan ${ }^{34}$. Il listait ainsi comme les principaux groupes de la région du Kham : Kangfan 康番 (Xikang minzu, qui comprend les Xifan et les Guzong), Han immigrés, Luoluo 倮倮, Moso 摩些, Lisu 栗粟, Luzi 潞子, Qiuyi 俅夷, entre autres groupes ${ }^{35}$.

Ces efforts déployés par une nouvelle génération d'historiens et d'ethnologues de mise en ordre de la diversité ethnique et culturelle du pays ne s'arrêtèrent pas avec la politique de consolidation des frontières menée par le Guomindang. Ils culminèrent après la fondation de la République populaire de Chine avec la campagne d'《identification des minzu » (minzu shibie 民族识别) lancée dans les années cinquante. En effet, comme Thomas Mullaney en retrace l'histoire, dans l'urgence de la tenue du Congrès national du peuple prévu pour la fin de l'année i954, il fallait connaître la composition ethnique du pays, "faire le tri » parmi les dénominations existantes, pour être à même de sélectionner les représentants de chaque peuple non-han invités à y siéger. Un recensement fut donc organisé et le projet d'identification des minzu dû être mené à bien au cours d'une période extrêmement brève de juillet 1953 à mai $1954^{36}$. Face au grand nombre des prétendants au statut de minzu et à la pléthore d'ethnonymes recueillis par les équipes d'enquêtes du projet d'identification (plus de 200 pour la seule province du Yunnan), il fallait sélectionner les meilleurs candidats. Si ces choix furent contraints par les quatre critères énoncés par Staline, qui constituaient en principe la référence théorique déterminant ce qu'est un minzu (nation, nationalité), il y eut dans bien des cas des considérations plus pragmatiques ${ }^{37}$.

33. La province du Xikang fut créée en 1939 pour être ensuite démantelée en 1955. Elle incluait la plupart des régions tibétaines du Kham ; voir par exemple Fabienne Jagou, « Vers une nouvelle définition de la frontière sino-tibétaine : la Conférence de Simla (1913-1914) et le projet de création de la province de Xikang ", Extrême-Orient, Extrême-Occident, n ${ }^{\circ}$ 28, 2006, p. I47-167.

34. Ren Naiqiang, Xikang tujing 西康图经, dans Ren Naiqiang zangxue wenji 任乃强藏学 文集, vol. I, Beijing, Zhongguo zangxue chubanshe, 2009, p. 205-210.

35. Ren Naiqiang, Xikang tujing 西康图经, p. 208-209. On trouve dans cette liste les principaux groupes connus sous ces mêmes noms (avec des orthographes différentes) depuis la dynastie Yuan, tels qu'ils figurent par exemple dans le Yuan yitong zbi 元一统志 (Monographie générale des Yuan) : "Dans le circuit de Lijiang, il existe huit sortes de Barbares, lesquels se nomment Moso, Bai, Luoluo, Dongmen, Echang, Qiao, Tufan, Lu » (丽江路, 蠻有八種, 曰磨些, 曰白, 日罗落, 曰冬悶, 曰峨唱, 曰撞, 曰吐蕃, 曰卢). Cf. You Zhong, Yunnan minzu shi, p. 310. Le caractère $\mathrm{Lu}$ 卢 (潞, etc.) désignait les habitants de la vallée de la Salouen (et est généralement associé aux Lisu, et/ou actuels $\mathrm{Nu}$ ), ce fleuve étant alternativement appelé $\mathrm{Lu}(\mathrm{zi}$ )jiang 潞(子)江 ou Nujiang 怒江. C'est aussi la première apparition du terme Qiao 撞 qui réapparaît dans des sources plus tardives sous la forme Qiu 俅.

36. Sur les conditions de réalisation du recensement, voir T. Mullaney, Coming to Terms.

37. Voir l'introduction à ce volume pour une discussion du concept de minzu, et des quatre critères de Staline : une langue, un territoire, un mode de production économique communs, et une même culture. L'ethnologue Lin Yaohua avait déjà souligné que ce fut souvent le critère 
L'héritage républicain joua alors un rôle important, et la classification ethnique sur des bases de classification linguistique s'imposa comme la méthodologie dominante utilisée par les ethnologues chinois, en continuité avec les pratiques des années trente et quarante. Les groupes linguistiques devinrent ainsi des substituts de l'ethnicité, et les taxinomies s'organisèrent souvent sur la base des divisions entre langues et groupes dialectaux ${ }^{38}$. Une comparaison entre la classification de $\mathrm{H}$. R. Davies et celles des chercheurs de l'ère républicaine tels que l'historien $\mathrm{Ma}$ Changshou, l'archéologue et géologue Ding Wenjiang 丁文江 (I884-1936), l'ethnologue Ling Chunsheng 凌純聲 (1902-198I), et le linguiste Luo Changpei 羅常培 (I899-1958), aboutit à des listes quasiment identiques dont dérivent la plupart des minzu actuels (voir tableau I ci-dessous) ${ }^{39}$.

Tableau 1 : Ancienne classification des sous-groupes de langue tibéto-birmane de la bordure sino-tibétaine, et ethnonymes associés ${ }^{40}$.

\begin{tabular}{|c|c|c|c|c|c|c|}
\hline $\begin{array}{l}\text { H.R. } \\
\text { Davies } \\
\text { (1909) }\end{array}$ & $\begin{array}{l}\text { Ding } \\
\text { Wen- } \\
\text { jiang } \\
\text { (1935) }\end{array}$ & $\begin{array}{l}\text { Ma } \\
\text { Changshou } \\
(1936)\end{array}$ & $\begin{array}{l}\text { Ling } \\
\text { Chun- } \\
\text { sheng } \\
\text { (1936) }\end{array}$ & $\begin{array}{l}\text { Luo } \\
\text { Changpei } \\
\text { et Fu Maoji } \\
\text { (1954) }\end{array}$ & $\begin{array}{l}\begin{array}{l}\text { Liste du } \\
\text { recense- } \\
\text { ment }\end{array} \\
1953-1954\end{array}$ & $\begin{array}{l}\text { Minzu } \\
\text { actuels }\end{array}$ \\
\hline \multicolumn{7}{|c|}{ I. Groupe tibétain } \\
\hline Tibétain & Zang & $\begin{array}{l}\text { Zang } \\
\text { Guzong }\end{array}$ & $\begin{array}{l}\text { Zang } \\
\text { Guzong } \\
\text { (Yun- } \\
\text { nan) }\end{array}$ & Zang & $\begin{array}{l}\text { Zang } \\
(668 \mathrm{r} 6 \\
\text { pers.) }\end{array}$ & $\begin{array}{l}\text { Zang } \\
\text { 藏族 }\end{array}$ \\
\hline \multicolumn{7}{|c|}{ 2. Groupe Hsi-fan (Xifan) } \\
\hline Xifan & Xifan & Xifan & Xifan & Xifan & $\begin{array}{l}\text { Xifan } \\
\text { (I6 o9I } \\
\text { pers.) }\end{array}$ & $\begin{array}{l}\text { Zang } \\
\text { 藏族 } \\
\text { Pumi } \\
\text { 普米族 }\end{array}$ \\
\hline Naxi & -- & Moso & $\begin{array}{l}\text { Naxi } \\
\text { (Moso) }\end{array}$ & Naxi & $\begin{array}{l}\text { Naxi } \\
\text { (I4I } 727 \\
\text { pers.) }\end{array}$ & $\begin{array}{l}\text { Naxi } \\
\text { 納西族 }\end{array}$ \\
\hline $\begin{array}{l}\mathrm{Nu} \\
\text { (A-nung) }\end{array}$ & $\mathrm{Nu}$ & $\mathrm{Nu}$ & $\mathrm{Nu}$ & $\mathrm{Nu}$ & $\begin{array}{l}\mathrm{Nu} \\
\text { (13 } 003 \\
\text { pers.) }\end{array}$ & $\begin{array}{l}\mathrm{Nu} \\
\text { 怒族 }\end{array}$ \\
\hline
\end{tabular}

linguistique qui prima dans le processus d'identification. Voir Lin Yaohua 林耀华, «Zhongguo Xinan diqu de minzu shibie »中国西南地区的民族识别 (L'identification des minzu dans le SudOuest de la Chine), dans Yunnan sheng bianji zu 云南省编辑组, éd., Yunnan shaoshu minzu shebui lishi diaocha ziliao buibian 云南少数民族社会历史调查资料汇编, vol. III, Kunming, Yunnan minzu chubanshe, I987, p. I-6.

38. Voir T. Mullaney, "Ethnic Clasification Writ Large. The 1954 Yunnan Province Ethnic Classification Project and Its Foundations in Republican-Era Taxonomic Thought," China Information, vol. I8, $\mathrm{n}^{\circ} 2$, 2004, p. 207-24I ; et Coming to Terms.

39. Ibid., p. 58-68.

40. Sources: T. Mullaney, Coming to Terms, tableau 7 et 8 p. 6o, 66-67 ; appendice C, p. I42-I44; Ma Changshou, «Zhongguo Xi'nan minzu fenlei », p. $64-65$. 


\begin{tabular}{|c|c|c|c|c|c|c|}
\hline \multicolumn{7}{|c|}{ Groupe Lolo } \\
\hline Lo-lo & Luoluo & Luoluo & Luoluo & Yi & $\begin{array}{l}\text { Yi } \\
\text { (I } 493347 \\
\text { pers.) }\end{array}$ & $\mathrm{Yi}$ 彝族 \\
\hline Lisu & Lisu & Lisu & Lisu & Lisu & $\begin{array}{l}\text { Lisu } \\
(24846 \\
\text { pers.) }\end{array}$ & $\begin{array}{l}\text { Lisu 傈 } \\
\text { 傈族 }\end{array}$ \\
\hline \multicolumn{7}{|c|}{ 4. Groupe birman } \\
\hline & & & Qiu & Qiu & $\begin{array}{l}\text { Qiu (non } \\
\text { renseigné) }\end{array}$ & $\begin{array}{l}\text { Dulong } \\
\text { 獨龍族 }\end{array}$ \\
\hline \multicolumn{7}{|c|}{ 5. $\quad--$} \\
\hline & & & & Qiang & $\begin{array}{l}\text { Qiang } \\
\text { (I pers.) }\end{array}$ & $\begin{array}{l}\text { Qiang } \\
\text { 姜族 }\end{array}$ \\
\hline
\end{tabular}

Dans cette tentative de reconfiguration de ce monde intérieur de la Chine, la plupart des chercheurs partageaient l'idée que la diversité des termes n'était pas représentative de la réalité, et que des regroupements étaient possibles et justifiés. La diversité culturelle et linguistique de la bordure sino-tibétaine en fit l'une des régions où les problèmes d'identification devinrent particulièrement épineux. Pour ne prendre que l'exemple des groupes déjà évoqués, l'on voit dans le tableau i que la classification linguistique servit de modèle pour la détermination des minzu, aboutissant dans certains cas à une simple révision de l'orthographe, ou rectification des noms ${ }^{41}$. Comme S. Harrell l'a déjà souligné, les «nouvelles » identifications réalisées dans les années cinquante aboutirent souvent à confirmer ou légitimer des distinctions pour beaucoup déjà présentes dans les catégories en usage avant la Libération ${ }^{42}$. La disparition de la catégorie Xifan témoigne en revanche d'un éventail d'arrangements contingents sur lesquels nous allons nous pencher plus loin.

\section{Histoire régressive et unité nationale}

Cette réorganisation a minima de la diversité ethnique du pays lors du projet de classification en minzu fut donc menée sur la base de considérations non seulement politiques, mais aussi linguistiques, ethnologiques et historiques soumises à l'intérêt national. Pour ce qui concerne la Chine du Sud-Ouest, les anthropologues

4I. Généralement ignoré, un antécédent à la rectification des dénominations fut le travail de Ruey Yih-fu (Rui Yifu 茌兔夫). Comme l'a relevé M. Fiskesjö, Ruey débuta en 1932 un vaste projet de rationalisation de l'ethnonymie à l'échelle du pays, avec en particulier pour objectif de rectifier les connotations dérogatoires des termes souvent attribués aux groupes périphériques. Voir M. Fiskesjö, "Rescuing the Empire: Chinese Nation-Building in the Twentieth Century," European Journal of East Asian Studies, vol. 5, n' 2, 2006, p. 15-44 ; et "The Animal Other: China's Barbarians and their Renaming in the Twientieth Century," Social Text, vol. 29, n' 4, 20II, p. 57-79.

42. S. Harrell, "The History of the History of the Yi," dans S. Harrell, éd., Cultural Encounters on China's Ethnic Frontiers, p. 66, 82. 
Lin Yaohua 林耀華 (I9I0-2000) et Fei Xiaotong 費孝通 (I9I0-2005), mais aussi le linguiste Fu Maoji 傅禁勣 (I9II-I988) et l'historien Fang Guoyu 方國瑜 (I903-I983) figuraient en première ligne.

Avant les années I950, de nombreux chercheurs tels que Fang Guoyu 方國瑜, Xu Jiarui 徐嘉瑞 (I895-1977) et Xiang Da 向達 (I900-I966) avaient commencé des recherches sur l'histoire des groupes ethniques du Yunnan ${ }^{43}$. Ils établirent des liens entre divers «barbares » du Sud-Ouest (Man ou Yi) et l'ancien ensemble des Di et Qiang (氏姜), justifiant une continuité historique entre les groupes identifiés à l'époque antique et ceux connus à l'époque moderne. Les travaux de Gu Jiegang, Ren Naiqiang et Ma Changshou concernant les Qiang et la reconstruction d'une histoire plurimillénaire s'inscrivent dans un même mouvement ${ }^{44}$. Il était assez bien établi à l'époque, comme R. Stein l'avait évoqué, de considérer les anciens Qiang comme les ancêtres non seulement des tibétains modernes ${ }^{45}$, mais aussi de bien d'autres groupes de langues tibéto-birmanes. Ces antécédents républicains relèvent en effet de l'effort d'une certaine intelligentsia à établir les bases d'une "unité du passé » pour les groupes amenés à participer à l'édifice national, soit sous la forme d'une ancestralité commune, soit par le biais de liens historiques étroits ${ }^{46}$.

L'historien Fang Guoyu, selon Ma Jianxiong, peut être considéré comme l'un des fondateurs de ce «paradigme académique » de la géographie historique du Yunnan et de l'histoire des nationalités minoritaires en Chine du Sud-Ouest particulièrement soucieuse des origines ${ }^{47}$. De telles recherches ont alimenté les perspectives empruntes d'évolutionnisme social appliqué à l'histoire des minorités chinoises, et devinrent en somme un outil académique au service de la stratégie politique visant à jeter les bases d'une histoire nationale unifiée, et à même de justifier le bien-fondé des catégories identifiées (les minzu officiels), en restituant leur développement au fil des siècles et leurs possibles origines (zu yuan 族源).

Ce paradigme des origines prend donc les catégories officiellement reconnues de nationalités comme point de départ, pour mettre en œuvre une histoire régressive dont la finalité est de les associer aux noms de groupes « ancestraux » figurant dans les textes historiques anciens. Selon cette approche - qui, faut-il le souligner, fait

43. Voir par exemple Xiang Da 向达, Man sbu jiaozbu 蛮书校注 (Le Manshu annoté), Beijing, Zhonghua shuju, 1962.

44. Voir Wang Mingke, Qiang zai Han-Zang zhi jian, p. 138-I42; et "From the Qiang Barbarians to the Qiang Nationality".

45. Voir, parmi d'autres, la discussion critique dans Gelei 格勒, Zangxue, renlei xue lunwen ji 藏學、人類學論文集 (Recueil d'écrits en tibétologie et anthropologie), vol. I, Beijing, Zhongguo zangxue chubanshe, 2008, p. 42-87.

46. Cette approche généalogique et raciale est très bien discutée par J. Leibold, Reconfiguring Chinese Nationalism. Pour une analyse de travaux de Ren Naiqiang dans cette optique, voir Tsomu Yudru, "Taming the Khampas: The Republican Construction of Eastern Tibet," Modern China, vol. 39, n ${ }^{\circ}$, 2013, p. 319-44.

47. Ma Jianxiong, "Local Knowledge Constructed by the State: Reinterpreting Myths and Imagining the Migration History of the Lahu in Yunnan, Southwest China," Asian Ethnology, vol. $68, \mathrm{n}^{\circ} \mathrm{I}, 2009$, p. III-I29. 
peu cas de l'histoire orale et des conceptions indigènes de l'ancestralitét ${ }^{8}$ — toutes les minorités actuelles seraient issues, in fine, d'une même origine. La tâche de construire une histoire des nationalités minoritaires a donc consisté en l'élaboration d'un arbre généalogique de leur évolution, remontant aux catégories aussi anciennes que celles de Qiang ou Di par exemple, et présumant de manière anachronique une continuité avec les groupes actuels - nous allons en voir plusieurs exemples.

C'est ainsi une mentalité généalogique qui, se déployant et incorporant les identités locales au sein d'histoires supra-locales, contribue à former le discours national sur l'origine et le développement historique de la Chine ${ }^{49}$. L'histoire régressive des minorités s'est imposée assez largement, amenant les intéressés à s'approprier cette aura d'ancestralité et à reformuler leurs mythes d'origine, et partant leur identitéso. Le plus souvent, l'identité de minzu se voit ainsi réaffirmée par la voix des membres éduqués en leur sein. Ce qui peut aboutir à une volonté de maintenir l'unité de son minzu face aux tentatives "séparatistes » de certains sous-groupes, ce que je vais aborder en décrivant plusieurs parcours menant à la consolidation de l'identité de minzu, malgré l'existence de négociations et de contestations.

\section{Destins taxinomiques}

Ce tableau de la diversité taxinomique nous conduit au devenir de certains des ethnonymes mentionnés précédemment, et aux résultats du processus d'identification et de classification qui aboutit à la reconnaissance des 55 nationalités minoritaires à l'échelle de la République populaire. Ce processus, mené dans l'urgence après l'avènement de la RPC, fut suivi d'enquêtes complémentaires jusque dans les années I980, pour réexaminer des revendications d'accès au statut de minzu, et dans un petit nombre de cas aboutir à de nouvelles reconnaissances ${ }^{51}$.

48. L'historien Shi Shuo 石硕 a cependant, avec d'autres, souligné la nécessité de prendre en compte les traditions orales et les acquis des recherches archéologiques ; voir Shi Shuo, «Zang-Yi zoulang diqu Zang-Mian yu minzu qiyuan wenti yanjiu pingshu»藏彝走廊地區藏緬語民族起源問 題研究評述, Xixiang zhanxian, vol. 34, $\mathrm{n}^{\circ}$ 2, 2008, p. I5-19.

49. Une collection telle que celle des Précis d'histoire de la littérature (wenxue jianshi 文學簡史) des nationalités minoritaires de Chine, devient nécessairement une histoire normative dont la chronologie est celle de l'histoire de la Chine et des dynasties successives. Voir ma discussion dans S. Gros, «Le lieu du pouvoir. Mémoire et enjeu de l'histoire chez les Drung du Yunnan (Chine), dans Gisèle Krauskopff, éd., Les faiseurs d'bistoires, Nanterre, Société d'ethnologie, 2009, p. 187-215.

50. K. Wellens, concernant deux groupes de la bordure sino-tibétaine (les Namuyi et les Premi), a montré comment cette internalisation de l'histoire officielle passe par un motif mythique reformulé et rediffusé de manière hégémonique. Voir K. Wellens, "Migrating Brothers and PartyState Discourses on Ethnic Origin in Southwest China," dans T. Huber et S. Blackburn, éds., Origins and Migrations in the Extended Eastern Himalayas, Leiden, Brill, 2012, p. 299-319. C'est aussi ce que démontre Ma Jianxiong à propos des Lahu du Yunnan dont une partie de l'élite éduquée a en somme "naturalisé » la version officielle de leur histoire et de leur origine ; voir Ma Jianxiong, "Local Knowledge Constructed by the State".

5I. Ainsi, Huang Guangxue 黄光学 et Shi Lianzhu 施联朱 identifient-ils quatre périodes dans le travail d'identification (1949-1954, 1954-1964, 1965-1978, 1978-1990), déterminées notamment 
Nous avons vu que les catégories linguistiques en usage influencèrent la taxinomie qui contribua à la détermination des catégories de minzu dans les années I950. Or, assez paradoxalement, si les catégories taxinomiques dérivées des classifications linguistiques tendaient à s'imposer, la langue en tant que telle n'était pas nécessairement un critère dominant. La catégorie Xifan constituait par exemple un tel ensemble linguistique, mais les connaissances relatives à sa composition et aux relations entre les langues qu'il réunissait étaient alors rudimentaires.

Ce que Fei Xiaotong a appelé le «couloir tibéto-yi » (Zang-Yi minzu zoulang) devint à la fin des années 1980 un champ privilégié pour les études linguistiques essentiellement sous l'impulsion du linguiste Sun Hongkai 孙宏开 ${ }^{52}$. Bien qu'encore discuté, cela a abouti à un redécoupage de la classification linguistique, et à la découverte de nouvelles langues. De sorte que les langues dites Xifan autrefois sont aujourd'hui reconnues pour la plupart comme un ensemble Qiangique au sein du groupe des langues sino-tibétaines, et des sous-ensembles rGyal-rong, Ersu, et $\mathrm{Na}$ sont également distingués. Ces ensembles regroupent notamment les Nameze (Namuyi 納木依), Minyak (Muya 木雅), Ersu 爾苏, rGyal-rong (Jiarong 嘉线), Horpa (Ergong 爾壟), Premi/Prinmi (Pumi 普米), Shixing 史興 (Xumi 旭米), nDra-pa (Zhaba 扎坝), Choyo (Queyu 却育), Lavrung, et Guiqiong 贵琼, divers groupes qui ont été intégrés au sein du minzu Zang (Tibétains). Plus au sud se trouvent par exemple les Nu et Dulong, dont la place au sein des sous-groupes de langues tibétobirmanes n'est pas encore bien établie mais qui, pour certains linguistes, pourraient être regroupés avec le rGyal-rong au sein d'une branche dite Rung ${ }^{\Im 3}$.

par les recensements nationaux ; voir Huang Guangxue et Shi Lianzhu, Zhongguo de minzu shibie: 56 ge minzu de laili 中國的民族識別— 56 各民族的來歷 (L'identification ethnique en Chine. L'origine des 56 nationalités), Beijing, Minzu chubanshe, 2005, p. I04-1I7. Voir également Wang Jianmin et al., Zhongguo minzu xue shi 中國民族學史 (Histoire de l'ethnologie chinoise), vol. 2 (1950-1997), Kunming, Yunnan jiaoyu chubanshe, I998, p. I06-I29. Précisons qu'il existe aujourd'hui en Chine tout un ensemble de «minzu non identifiés 》 (wei shibie minzu未識別民族) : certains groupes ayant été l'objet d'investigations mais dont la reconnaissance officielle et l'accès au statut de minzu furent ajournés (et le restera probablement), ou d'autres ayant été amalgamés au sein d'un seul minzu mais revendiquant une identité séparée sur la base de différents marqueurs identitaires. Trente-huit nationalités furent reconnues en 1953 ; quinze furent ajoutées en 1964; et les dernières reconnaissances eurent lieu en 1965 (Luoba) et 1979 (Jinuo). Dès lors, tous les autres cas de revendications aboutirent à la confirmation de la classification existante.

52. Son article fondateur est le suivant : Sun Hongkai, «Liujiang liuyu de minzu yuyan ji qi xishu fenlei 六江流域水文民族語言及其係數分類 ”, Minzu xuebao 民族學報, vol. 3, 1983, p. 196-213. Un grand nombre d'études ont été menées depuis, par Sun lui-même et d'autres linguistes, dont il est impossible de rendre compte ici.

53. Regroupant le T'rung (Dulong), le Rawang, avec le Kiranti, le Kham, et autres langues de l'Himalaya de l'ouest (Kinauri-Almora). Cf. Randy J. LaPolla, "Overview of Sino-Tibetan Morphosyntax," dans G. Thurgood et R. J. LaPolla, éds., The Sino-Tibetan Languages, Londres, Routledge, 2003 , p. 22-42. Les problèmes de classification au sein du groupe des langues dites tibéto-birmanes sont toujours d'actualité et donnent lieu à de nouveaux découpages. Voir notamment George van Driem, "The ancestry of Tibetan," dans Gray Tuttle, Kunsang Gya, Karma Dare et Johnathan Wilber, éds., The Third International Conference on Tibetan Language, Volume I: Proceedings of the Panels on Domains of Use and Linguistic Interactions, New York, Trace 


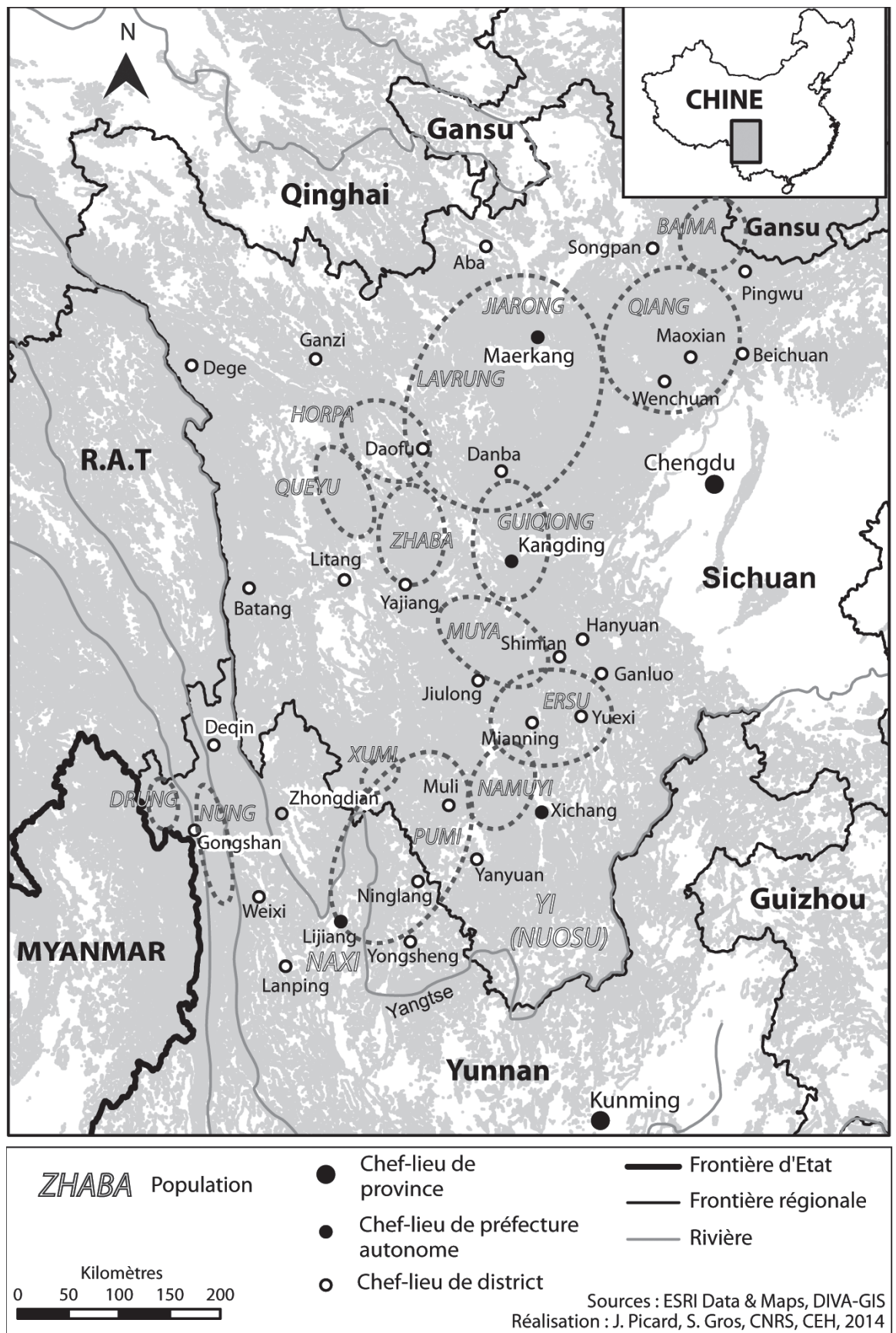

Carte 1 : Localisation des principaux groupes en présence dans les marches sinotibétaines. 
Le tableau 2 ci-dessous liste ces divers groupes qui peuplent la bordure du plateau Tibétain, en les organisant globalement dans une succession nord-sud (voir la carte I), et en indiquant leur identité de minzu, ainsi que les autonymes et noms conventionnels sous lesquels ils sont connus, notamment comme groupes linguistiques ${ }^{54}$.

Tableau 2 : Principaux groupes en présence dans les marches sino-tibétaines : noms et localisations.

\begin{tabular}{|c|c|c|c|}
\hline $\begin{array}{l}\text { Nom de } \\
\text { minzu }\end{array}$ & $\begin{array}{l}\text { Nom conventionnel } \\
\text { (autonyme) }\end{array}$ & Province & $\begin{array}{l}\text { Principales localisations } \\
\text { (unités administratives) }\end{array}$ \\
\hline Qiang 差 & $\begin{array}{l}\text { Qiang 姜 } \\
(r m a, r m a, z m a)\end{array}$ & $\begin{array}{l}\text { Gansu, } \\
\text { Sichuan }\end{array}$ & $\begin{array}{l}\text { Songpan 松潘縣, Heishui 黑水縣, } \\
\text { Maoxian 茂縣, Wenchuan 汶川縣, } \\
\text { Beichuan 北川縣, Lixian 理縣 }\end{array}$ \\
\hline \multirow[t]{5}{*}{ Zang 藏 } & $\begin{array}{l}\text { Baima Zangzu } \\
\text { 白馬藏族 } \\
\text { Dwags-po } \\
(p e)\end{array}$ & Sichuan & $\begin{array}{l}\text { Pingwu 平武縣, Nanping 南坪縣, } \\
\text { Wenxian 文縣, Songpan 松潘, } \\
\text { Jiuzhaigou 九寨沟 }\end{array}$ \\
\hline & $\begin{array}{l}\text { Jiarong 嘉线, rGyal-rong } \\
\text { - Situ 四土, Japhug, Tsho- } \\
\text { dun, Zbu } \\
\text { - Lavrung (Khroskyabs) } \\
\text { - Horpa, Ergong 爾龔 } \\
\text { (Royba, sTodpa, Japhug, } \\
\text { kə-ru, kuru, la vruy...) }\end{array}$ & Sichuan & $\begin{array}{l}\text { Ma'erkang 馬爾康縣, Daofu 道孚縣, } \\
\text { Danba 丹巴縣, Lixian 理縣 }\end{array}$ \\
\hline & $\begin{array}{l}\text { Guiqiong 贵琼 } \\
\left.\text { (gu t6 } 6^{h} \tilde{J}, g w i t f^{p} \circ \eta\right)\end{array}$ & Sichuan & Kangding 康定縣, Danba 丹巴縣 \\
\hline & $\begin{array}{l}\text { Zhaba 扎坝, 扎巴 } \\
\text { nDra-pa, (Tib.) 'Dra-pa } \\
\text { Choyo, Queyu 却育 } \\
\text { (ndza pa, }{ }^{n} \text { da pi) }\end{array}$ & Sichuan & $\begin{array}{l}\text { Daofu 道孚縣, Yajiang 雅江縣 } \\
\text { Xinlong 新龍縣 }\end{array}$ \\
\hline & 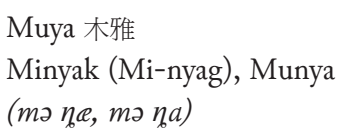 & Sichuan & $\begin{array}{l}\text { Jiulong 九龍縣, Kangding 康定縣, } \\
\text { Shimian 石棉縣 }\end{array}$ \\
\hline
\end{tabular}

Foundation, 2013, p. 363-397 ; et Guillaume Jacques et Alexis Michaud, "Approaching the Historical Phonology of Three Highly Eroded Sino-Tibetan Languages: Naxi, Na and Laze, Diachronica, vol. 28, $\mathrm{n}^{\circ}$ 4, 20II, p. 468-498.

54. Pour l'identification de ces diverses langues, voir par exemple Sun Hongkai, "Languages of the Ethnic Corridor in Western Sichuan" (R. J. LaPolla, trad.), Linguistics of the Tibeto-Burman Area, vol. I3, nº I, I990, p. I-31. Le travail ethnographique pionnier de Long Xijiang 龍西江 concernant les «Xifan » faisait déjà état de ces sous-groupes, aujourd'hui essentiellement distingués par le biais des recherches linguistiques ; voir K. Wellens, "What's in a Name?", p. 25. De nombreuses études linguistiques dont il est impossible ici de rendre compte ont désormais été menées sur ces langues. 


\begin{tabular}{|c|c|c|c|}
\hline & $\begin{array}{l}\text { Ersu 爾苏 }\left(\partial^{t}-s u, p u-\partial^{t}-z z\right) \\
\text { Lisu 栗苏 ou Liru 里汝 } \\
(l i-z u, l y-z u) \\
\text { Tosu (Tib. Tog-su), } \\
\text { Duoxu 多續 }(d o-6 u)\end{array}$ & Sichuan & $\begin{array}{l}\text { Ganluo 甘洛縣, Yuexi 越西縣, } \\
\text { Shimian 石棉縣, Hanyuan } \\
\text { 漢源縣, Jiulong 九龍縣, } \\
\text { District autonome tibétain de Muli } \\
\text { 木里藏族自治縣, Mianning 冕寧縣 }\end{array}$ \\
\hline & $\begin{array}{l}\text { Namuyi 納木依, 納木義, } \\
\text { Namuri 納木日 } \\
(n a-m z i, n a-m z \imath, \\
n \mathfrak{e}-m u-z i)\end{array}$ & Sichuan & $\begin{array}{l}\text { Mianning 冕寧縣, } \\
\text { District autonome tibétain de Muli } \\
\text { 木里藏族自治縣 }\end{array}$ \\
\hline & $\begin{array}{l}\text { Shixing 史興 } \\
\text { Xumi 旭米 } \\
\text { ( } u b \tilde{e}, \text { subi) }\end{array}$ & Sichuan & $\begin{array}{l}\text { District autonome tibétain de Muli } \\
\text { 木里藏族自治縣 }\end{array}$ \\
\hline Yi 彝 & $\begin{array}{l}\text { Nuosu 诺苏 } \\
(\Im \text { 拉 } n \bar{s} \bar{s} \bar{u})\end{array}$ & Sichuan & $\begin{array}{l}\text { Préfecture autonome Yi de } \\
\text { Liangshan 凉山彝族自治州 }\end{array}$ \\
\hline Pumi 普米 & $\begin{array}{l}\text { Premi, Prinmi } \\
\left(p^{b} r i n m i, \text { var. } p^{b} z \tilde{\partial}-m i,\right. \\
\left.p^{h} \measuredangle \tilde{o}-m \partial, t \hat{s}^{n} \tilde{\partial}-m i\right)\end{array}$ & $\begin{array}{l}\text { Sichuan, } \\
\text { Yunnan }\end{array}$ & $\begin{array}{l}\text { District autonome tibétain de Muli } \\
\text { 木里藏族自治縣, Yanyuan 盐源縣, } \\
\text { Jiulong 九龍縣, au Sichuan } \\
\text { Lanping 蘭坪縣, Ninglang 寧蒗 } \\
\text { 彝族自治縣, Yongsheng 永勝縣, } \\
\text { Yunxian 雲縣, Weixi 維西縣, } \\
\text { Lijiang 麗江納西族自治縣 }\end{array}$ \\
\hline $\begin{array}{l}\text { Naxi 納西 } \\
\text { Mengu } \\
\text { 蒙古 }\end{array}$ & $\begin{array}{l}\text { Na } \\
\text { Mosuo 摩梭 } \\
\text { (Nabin, Naru, Laze, } \\
\text { Rek'ua) }\end{array}$ & Yunnnan & $\begin{array}{l}\text { Lijiang 麗江納西族自治縣, } \\
\text { Yongning 永寧縣, Yanyuan 鹽源縣, } \\
\text { Yongsheng 永勝縣, Yunxian 雲縣, } \\
\text { Weixi 維西縣, Ninglang 寧蒗彝族 } \\
\text { 自治縣, Zhongdian 中甸縣, } \\
\text { Huaping 華坪縣, Deqin 德欽縣, } \\
\text { Mangkang 芒康縣, Batang 巴塘 }\end{array}$ \\
\hline $\begin{array}{l}\text { Dulong } \\
\text { 獨龍 }\end{array}$ & $\begin{array}{l}\text { Drung } \\
(\text { toruy })\end{array}$ & Yunnan & $\begin{array}{l}\text { District autonome Drung et } \mathrm{Nu} \text { de } \\
\text { Gongshan 貢山獨龍族怒族自治縣 }\end{array}$ \\
\hline $\mathrm{Nu}$ 怒 & 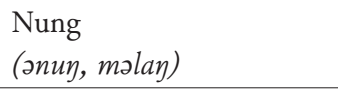 & Yunnan & $\begin{array}{l}\text { District autonome Drung et } \mathrm{Nu} \text { de } \\
\text { Gongshan 貢山獨龍族怒族自治縣 }\end{array}$ \\
\hline
\end{tabular}

Note : Cette liste n'est pas exhaustive, et ne sont pas mentionnés les Han, Tibétains (Zang) dans leur ensemble, catégories majoritaires et inclusives régionalement. Les localisations sont données à titre indicatif, et ne font pas état de la dispersion des groupes listés.

(C) École française d'Extrême-Orient, Paris, 20I5

Do not circulate without permission of the editor / Ne pas diffuser sans autorisation de l'éditeur 
L'ensemble des groupes pris en considération ici sont ceux localisés directement sur la bordure orientale du plateau tibétain (voir carte I), et ayant été plus particulièrement l'objet d'influences culturelles, religieuses ou politiques venant du Tibet. Le tableau 2 montre la diversité linguistique, désormais mieux connue, au sein de la catégorie Zang, qui permet de distinguer plusieurs groupes dont les différences se retrouvent également sur le plan culturel. Ceux-ci, autrefois reconnus comme Xifan, furent dans leur grande majorité intégrés au minzu Zang essentiellement sur la base de similarités liées à l'influence de la culture et de la religion tibétaines, et à l'usage courant localement du tibétain comme lingua franca. L'ensemble des groupes reconnus comme Zang attestent de divers degrés de tibétanisation. Ainsi, suivant Koen Wellens, si l'on cherchait à établir un continuum du degré d'intégration à la société et la culture tibétaines, les Namuyi seraient à l'extrême de la moindre intégration, et les Minyak et rGyal-rong à l'autre extrémité, avec les Pumi (Premi) quelque part au milieu ${ }^{\varsigma 5}$. Plus au Sud, les Dulong (Drung) et $\mathrm{Nu}$ (Nung), bien que relativement tibétanisés pour ces derniers, sont restés à la marge de l'expansion tibétaine et furent toujours considérés comme non-tibétains en raison de différences socio-culturelles marquées. Les Naxi/Mosuo ont de longue date entretenu des rapports commerciaux, religieux et politiques étroits avec les Tibétains du Kham, tout en ayant maintenu un fort pouvoir régional. Enfin, les Yi (Nuosu) constituaient pour leur part une sorte de limite orientale de l'influence tibétaine.

Parmi les groupes identifiés comme Zang et ceux qui bénéficient d'un statut de minzu à part entière - tels les Qiang autrefois considérés comme Xifan — s'ils ont tous entretenu des liens historiques étroits avec les sociétés tibétaines du Kham, divers agencements pragmatiques aboutirent aux identifications actuelles. Rappelons par exemple que le terme Qiang n'apparaissait pas parmi les ethnonymes recensés lors de la première vague de la campagne d'identification (cf. tableau I, supra), ni parmi les catégories linguistiques alors en usage (car intégré à l'ensemble Xifan). Cette appellation qui existe depuis la dynastie des Shang 商 (I250-IO5O av. J.-C.) $)^{56}$, et qui en vint à désigner un ensemble complexe et changeant de populations barbares de l'Ouest, fut en somme ressuscitée, «fossile vivant » de l'ethno-taxinomie. Si pendant la période de la campagne d'identification, les intéressés s'étaient pour certains fait recenser comme Zang (Tibétains), d'autres comme Qiang, voire encore comme Han, c'est la catégorie Qiang qui s'est imposée officiellement, et est devenue le nom officiel de cette «nationalité minoritaire » à laquelle la plupart des intéressés se sont ralliés au dépend de l'usage de leur autonyme, Rma. Comme le souligne justement Wang Mingke, cette variabilité de l'identification est intéressante, non pas tant parce qu'elle souligne la contingence des catégories de minzu, que parce qu'elle révèle la porosité des catégories ethniques utilisées localement, et les possibles fluctuations d'identification ${ }^{57}$. Quant aux autres groupes désignés par le label Xifan,

55. K. Wellens, "Migrating Brothers and Party-State Discourses," p. 307.

56. Les inscriptions oraculaires sur os font référence aux Qiang en tant que victimes sacrificielles ; voir Gideon Shelach, "The Qiang and the Question of Human Sacrifice in the Late Shang Period," Asian Perspectives, vol. 35, n I, 1996, p. I-26.

57. Wang Mingke, Qiang zai Han-Zang zhi jian, p. 65. 
ils eurent des destins ethnonymiques différents et certainement caractéristiques des aléas de l'assignation identitaire à l'époque. En fait, Xifan fit tout d'abord partie des ethnonymes conservés lors de la campagne d'identification, mais il fut proposé qu'il soit assimilé à la catégorie Zang (Tibétains). Comme nous allons le voir, certains de ces groupes maintenant mieux identifiés sur le plan linguistique peuvent avoir une position ambivalente quant à leur inclusion au sein de l'entité Zang.

Il semble nécessaire d'insister sur le fait que les catégories ethniques n'étaient pas autrefois nécessairement des référents significatifs, et que les distinctions intergroupes sur les bases de marqueurs culturels, religieux voire linguistiques, ne se faisaient donc pas sur les mêmes critères qu'aujourd'hui. Le langage de l'ethnicité s'est modifié en fonction du contexte dans lequel il s'est développé en RPC. De cette nouvelle configuration émergent différentes manières d'être ethnique en Chine selon les spécificités locales et les termes du dialogue qui peut prendre place. Plusieurs cas de figure ont été exposés par S. Harrell concernant les Nuosu, Prmi et Naze, avec pour chaque cas la dominance d'un mode d'expression identitaire : primordialiste, instrumental, ou contingent $t^{58}$. Il semble cependant difficile de distinguer ces différents modes qui peuvent être présents simultanément selon les acteurs pris en considération, et le discours public ou privé. Je vais ainsi exposer plusieurs cas d'identités négociées, dressant un panorama de la diversité des cas de figure le long de la bordure tibétaine et de la manière dont s'entremêlent les questions liées à l'origine, les distinctions de langue, et la relation aux catégories et classifications officielles.

\section{Retour sur les origines}

Le discours académique dominant en Chine concernant l'origine et les migrations des peuples du Sud-Ouest s'est désormais imposé même au-dehors des frontières nationales. Pour ne prendre que l'exemple de la nationalité Naxi, et du sous-groupe Mosuo 摩梭 (dérivé de l'ethnonyme plus ancien mentionné précédemment), il est courant, même en dehors de la RPC, de trouver des affirmations telles que celles de Shih Chuan-kang qui, en référence au travail pionnier de Fang Guoyu concernant l'origine des Naxi, affirme, malgré quelques réserves, qu'il existe une continuité culturelle et ethnique depuis l'époque où l'on trouve les premières mentions des Mo-sha ou Mo-so dans les annales chinoises ${ }^{59}$. Ou encore Guo Xiaolin qui écrit que « les Mosuo habitant aujourd'hui les piedmonts de l'Himalaya oriental sont les descendants du Royaume des Femmes (Nüguo), originellement une tribu Qiang du plateau Tibet-Qinghai $»^{60}$. Des affirmations péremptoires qui contrastent cependant avec les versions locales des récits d'origine, ou même les nuances et les doutes

58. S. Harrell, Ways of Being Ethnic.

59. Shih Chuan-kang, Quest for Harmony. The Moso Traditions of Sexual Union and Family Life, Stanford, Stanford University Press, 20IO, p. 23-24, 36-39.

6o. Guo Xiaolin. State and Ethnicity in China's Southwest, Leiden, Brill, 2008, p. 89. 
exprimés par divers historiens en Chine même, évoquant pour les Naxi trois principales origines possibles ${ }^{61}$.

Cette histoire régressive, pour problématique qu'elle soit, n'est pas sans points communs avec les traditions orales des peuples concernés. En effet, dans les deux cas il est fait référence à une terre ancestrale originelle et à des routes migratoires menant à la région d'habitat actuelle, ainsi qu'à l'idée d'une continuité ethnique avec ces ancêtres. La relation entre les deux est cependant ambiguë. Ainsi, dans le cas des Naxi pour continuer sur un même exemple, les idées relatives à la migration sont un support à une forme d'harmonisation des discours indigènes et nationaux : au premier se formulant dans les termes de la parenté et d'une géographie sacrée (les âmes des défunts suivent à rebours les routes migratoires pour retourner à la terre des ancêtres), répond le second dans les termes d'une vision unitaire de la diversité ethnique émanant d'une même source (les Qiang de la Chine ancienne) ${ }^{62}$.

La tradition tibétaine, évoquée en introduction à cet article, que R. Stein avait exploré, comporte de nombreux points de chevauchement avec les traditions des groupes voisins, Qiang, Naxi (Mosuo), Pumi (Premi), Yi (Nuosu), Ersu et d'autres encore : on y retrouve notamment les thèmes de la descente du ciel (par une corde ou une chaîne) sur une montagne ; la naissance de l'humanité d'un œuf primordial ; l'existence de quatre ou six clans primordiaux; le peuplement à partir d'un couple de germains (issu d'un singe et d'une démone), ou encore le thème des deux frères à partir desquels s'instaurent une différentiation ethnique. Autant de thèmes qui contribuent dans certains cas à alimenter un sentiment d'origine commune, et peuvent être utilisés à des fins de revendication identitaire.

L'histoire officielle, nourrie de l'histoire locale, peut être à son tour appropriée, sélectivement ou non, tout comme l'est l'identité de minzu associée à cette histoire. Concernant deux groupes de la bordure sino-tibétaine, les Namuyi et les Premi, $\mathrm{K}$. Wellens a montré comment l'internalisation de l'histoire officielle au sein de laquelle ils sont considérés comme Tibétains (Zang) passe par le motif mythique des deux

6I. Descendants des anciens Qiang 姜, Yi 夷, ou Tibétains 藏. L'interprétation d'une origine Qiang s'est certes imposée chez de très nombreux auteurs. Généralement attribuée à Fang Guoyu, elle fut en fait déjà formulée par Zhang Taiyuan 章太炎 dans son Xi'nan shuyi xiaoji 西南屬夷小記 (1936). Voir Shi Shuo, «Zang-Yi zoulang diqu ». Pour une histoire des Naxi dans leurs relations aux Tibétains, voir plus particulièrement Yang Fuquan 杨福泉, Naxizu yu Zangzu lishi guanxi yanjiu 纳西族与藏族历史全系研究 (Étude des relations historiques entre Naxi et Tibétains), Beijing, Minzu chubanshe, 2005 ; et Zhao Xinyu 赵心愚, Naxizu yu Zangzu guanxi sbi 纳西族与藏族 矢系史 (Histoire des relations entre Naxi et Tibétains), Chengdu, Sichuan renmin chubanshe, 2004. Voir également, pour une reconstruction de l'origine des Naxi et Mosuo, Christine Mathieu, History and Anthropological Study of the Ancient Kingdoms of the Sino-Tibetan Borderland: Naxi and Mosuo, Lewiston, The Edwin Mellen Press, 2003, chapitre 9 en particulier.

62. Voir C. McKhann, "Sacred Trails: Genealogical Mapping and the Creation of Historical space among the Naxi of Southwest China," Histoire et Anthropologies, Asies 2, 2003, p. 29-47. 
frères, reformulé de sorte à leur assigner désormais des ancêtres tibétains ${ }^{63}$. Par contraste, un cas exemplaire d'appropriation sélective est celui des intellectuels yi qui réécrivent à rebours leur histoire, certes en donnant alors à la catégorie officielle Yi une saillance incontestable, mais en renversant la situation à leur avantage et en se plaçant à l'origine de la civilisation chinoise ${ }^{64}$. Ce sont bien entendu les élites des nationalités minoritaires qui sont plus particulièrement en mesure d'affirmer par eux-mêmes leur identité de minzu, et selon leurs propres termes ${ }^{65}$.

Si lesdits Xifan localisés au Sichuan furent généralement reconnus comme Zang, les Xifan de la province du Yunnan (des régions de Lanping, Ninglang, et Lijiang) demandèrent et obtinrent une reconnaissance en tant que nationalité à part entière appelée Pumi 普米 ${ }^{66}$. Ce cas est tout à fait similaire à celui relativement mieux connu des Mosuo qui, en fonction du côté de la frontière où ils se trouvaient, furent soit regroupés au sein de la catégorie Naxi (au Yunnan), soit identifiés comme Menggu (Mongols), se revendiquant descendants de soldats de la dynastie des Yuan (au Sichuan). Aussi ces «mongols » de Dapo ou Wodi parlent-ils une langue tibétobirmane, et s'autodésignent-ils Naze, comme leurs « cousins » de nationalité Naxi du Yunnan ${ }^{67}$.

63. Voir K. Wellens, "Migrating Brothers and Party-State Discourses". Concernant l'identification des Pumi, voir également S. Harrell, "The Nationalities Question and the Prmi Prblem [sic]," dans M. J. Brown, éd, Negotiating Ethnicities in China and Taiwan, Berkeley, University of California Press, p. 274-296.

64. S. Harrell et Li Yongxiang, "The History of the History of the Yi, Part II," Modern China, vol. 29, n 3,2003 , p. 362-96.

65. Ainsi, selon $\mathrm{S}$. Harrell et $\mathrm{Li}$, « [Les] élites des minorités ont développé des versions contredisant le récit national, des versions dans lesquelles leur propre groupe ethnique ne fait plus nécessairement que contribuer à la couleur locale. Leurs versions du récit national questionnent activement la centralité de ce qu'ils considèrent comme la culture Han dans le développement de la nation chinoise. [...] Ce discours alternatif nait également de la consolidation de l'identite de groupe au niveau du minzu [...] ", dans "The History of the History of the Yi, Part II," p. 364.

66. Voir Shi Lianzhu et Huang Guangxue, Zhongguo de minzu shibie, p. 198 ; K. Wellens, "What's in a name? The Premi in Southwest China and the consequences of defining ethnic identity," Nations and Nationalism, vol. 4, n I, 1998, p. 17-34; T. Mullaney, Coming to terms, tableau I6, p. I18, p. I29. Chuan-kang Shih, Quest for Harmony, p. 43, mentionne l'usage encore courant de Xifan dans la région de Yongning au Yunnan pour faire référence aux Pumi (Premi). Concernant les Ersu, Wu Da évoque l'usage de la désignation Fanzu 番族 jusque dans les années 1990 ; voir Wu Da 巫達, Rentong zhi jueze: Sichuan Ersu ren zuqun rentong jiangou de minzu zhi yanjiu 认同之抉择一一四川尔苏人族群认同建构的民族志研究 (Choix d'identités. Recherche ethnographique sur la construction de l'identité ethnique des Ersu du Sichuan), Thèse de doctorat, Chinese University of Hong Kong, 2004.

67. Voir plus particulièrement Harrell, Ways of Being Ethnic, p. 216 et suiv.

(C) École française d'Extrême-Orient, Paris, 2015

Do not circulate without permission of the editor / Ne pas diffuser sans autorisation de l'éditeur 


\section{De la langue à l'ethnicité}

Au-delà d'une variété des parlés tibétains, considérés souvent comme dialectes ou langues à part entière ${ }^{68}$, il existe aujourd'hui 39 langues minoritaires identifiées au sein de la tibétosphère, dont certaines ont un nombre de locuteurs très limité. Comme Gerald Roche l'a récemment souligné avec raison, la diversité interne à la catégorie Zang émerge plus particulièrement si l'on se penche sur la diversité linguistique $^{69}$. Ainsi le long de la bordure sino-tibétaine, nous avons vu précédemment que plus d'une dizaine de sous-groupes Zang parlent des langues non-tibétaines. Ils sont ainsi minorité à double titre, en tant que minorité Zang de Chine, et en tant que minorité linguistique au sein de cette "nationalité » Zang. Ceci est particulièrement significatif, puisqu'en tant que tels, ils sont à même de recevoir une partie de leur éducation en tibétain, ce qui contribue au processus de tibétanisation, parallèle — sinon de même ampleur — à celui de sinisation ${ }^{70}$.

Le linguiste Sun Hongkai avait déjà souligné qu'une même "nationalité » (minzu) pouvait rassembler des locuteurs de plusieurs langues. Il donnait entre autres l'exemple des Jiarong, Ergong, Muya, Namuzi, etc., qui se seraient fondus, à la suite d'influences politiques et culturelles, à l'entité « Tibétaine », et selon lui « ne souhaitent pas être reconnus comme de nouvelles nationalités », tout comme d'autres qui parlant des langues qiang ou pumi souhaiteraient, selon lui, « être reconnus comme tibétains $»^{7^{\top}}$.

Ainsi, un peu à la manière dont la catégorie linguistique « tibéto-birmane » peut être utilisée confusément comme équivalent d'une catégorie ethnique, la catégorie Zang peut difficilement être appréhendée comme une catégorie ethnolinguistique qui impliquerait de facto un sentiment de cohésion. Les Xumi (Shixing) et les Baima sont par exemple deux cas contrastés révélateurs de la manière dont les identités locales s'accommodent de l'identité de minzu qui leur fut assignée, le critère linguistique étant relégué au second $\operatorname{plan}^{72}$. Si les deux groupes ont été classifiés comme Zang (Tibétains), les Xumi conçoivent désormais leur identité en des termes qui se conforment avec cette classification officielle, tandis que les Baima revendiquent une identité propre. Du point de vue linguistique, $\mathrm{K}$. Chirkova a souligné que si ces deux groupes, Xumi (Shixing) et Baima, parlent des langues significativement différentes des variétés de tibétain parlées alentours, la langue Xumi se rattache

68. Pour ce qui est des dialectes tibétains, voir plus particulièrement Nicolas Tournadre, "L'aire linguistique tibétaine et ses divers dialectes », Lalies, Paris, Presses de l'ENS, Éditions Rue d'Ulm, n' 25,2005 , p. 7-56.

69. G. Roche, "The Vitality of Tibet's Minority Languages in the Twenty-First Century," Multiethnica 35, 2014, p. 24-33.

70. Ibid., p. 27-28, mentionne aussi le fait que le tibétain s'impose comme lingua franca, et que les langues minoritaires des régions tibétaines sont généralement considérées en termes péjoratifs, comme "langues de fantômes » ('dre skad) ou "langues arriérées » (log skad).

7I. Voir Sun Hongkai, "Language Recognition and Nationality," International Journal of the Sociology of Language, vol. 97, $\mathrm{n}^{\circ}$ I, 2009, p. 9-22.

72. Récemment bien documenté par K. Chirkova, "Between Tibetan and Chinese." Le cas des Baima est désormais bien connu, et a été l'objet de nombreux écrits notamment en Chine même. 
plutôt aux langues tibéto-birmanes du sous-groupe qiangique, tandis que le Baima partage avec les dialectes tibétains de l'Amdo du vocabulaire et certains traits de syntaxe. Ce contraste révèle que la langue joue un rôle mineur dans l'affirmation d'une ethnicité, puisque contrairement aux Xumi, les Baima revendiquent une identité distincte et remettent en question leur inclusion dans la catégorie Zang (Tibétains) ${ }^{73}$. De toute évidence, la construction d'une appartenance ethnique ne peut pas être simplement réduite à une corrélation univoque avec la langue parlée, et les facteurs culturels et religieux jouent un rôle considérable.

Il est important de relever qu'à travers toute la région du Kham existe un grand nombre de dialectes qui ne sont pas mutuellement intelligibles. L'incontournable réalité de cette diversité des parlés aboutit à une grande tolérance à la diversité linguistique dans la région. Dans certains cas, les locuteurs de tels dialectes sont conscients qu'ils ne parlent pas tibétain, même s'ils se disent tibétains culturellement, tandis que d'autres n'ont pas vraiment l'idée que la langue qu'ils parlent est fondamentalement différente du tibétain ${ }^{74}$.

Au nord-ouest du Yunnan, le cas des Nuzu 怒族 et de ceux alors connus sous le nom de Qiu (zi) 俅(子) illustre d'une autre manière le caractère contingent de la détermination des catégories de minzu quant à ce qui devrait être une correspondance entre langue et identité. Les premières enquêtes semblaient révéler que ces deux groupes de la région de Gongshan devaient être réunis en une seule "nationalité », car ils se présentaient comme une même communauté économique, socioculturelle et linguistique ${ }^{75}$. Si la catégorie $\mathrm{Nu}$ en tant que telle regroupait des langues mutuellement inintelligibles, elle pouvait néanmoins, de l'avis des enquêteurs, constituer une catégorie pertinente, car une langue commune existait : la lingua franca qu'était le Lisu, langue de l'ethnie dominante localement. Pour compliquer la composition de ce groupe $\mathrm{Nu}$, dont la diversité linguistique fut ainsi rationalisée, les Qiu furent jugés suffisamment proches pour y être adjoints, et il fut alors provisoirement proposé que ce nouveau groupe, faute d'avoir un nom, pourrait être appelé «Luzu » 珞族 (nom réutilisant l'ancien caractère $\mathrm{Lu}$ des annales chinoises) ${ }^{76}$. Il fut finalement reconnu une nationalité Dulong (Dulongzu 獨龍族), distincte d'une nationalité Nu (Nuzu 怒族), laquelle perpétua l'identification d'un ensemble cependant hétérogène (par sa diversité linguistique et culturelle) dans son principal lieu d'habitat, qui au cours d'un long

73. K. Chirkova, "Between Tibetan and Chinese." L'auteure souligne sur ce point que la ligne officielle en Chine consiste à dire qu'il est possible que pour des raisons historiques, plusieurs langues (même génétiquement distinctes) soient parlées au sein d'un même minzu. On peut constater, encore une fois, combien ces ajustements pratiques relativisent l'application des quatre critères d'identifications dérivés des écrits de Staline sur la nation.

74. Je remercie Katia Chirkova et Guillaume Jacques pour avoir souligné ces aspects (communications personnelles). Il existe une opposition assez générale dans les milieux intellectuels tibétains contre le fait de considérer les langues dites qiangiques comme autre chose que du tibétain.

75. Yunnan sheng bianji zu 云南省编辑组, éd., Yunnan shaoshu minzu shehui lishi diaocha ziliao buibian 云南少数民族社会历史调查资料汇编 (Compilation de matériaux d'enquêtes sur l'histoire et la société des nationalités minoritaires du Yunnan), vol. III, Kunming, Yunnan minzu chubanshe, I987, p. 48-5I.

76. Cf. T. Mullaney, Coming to Terms, p. Io9-ıı. 
développement historique, se serait progressivement amalgamé (ronghe 融合) pour devenir une même communauté (gongtongti 共同體) 77 .

À l'heure actuelle, les différences dialectales significatives n'empêchent pas une communication transethnique entre $\mathrm{Nu}$ et Dulong, voire même avec les Rawang de l'autre côté de la frontière, en Birmanie. En revanche, au sein du seul minzu Nu, le sous-groupe Zauzou parle une langue incompréhensible des autres sous-groupes. Dans les deux cas, les sphères d'identité liées à la langue, l'ethnicité, et le minzu ne sont pas congruentes. En somme, il importe d'insister sur le fait que les valeurs culturelles et la force politique attachées aux langues sont dynamiques, contextuelles et changeantes, et les possibles variations d'identités ethnolinguistiques sont une réalité à prendre en compte.

\section{Le choix et l'assigné}

Dans son incontournable article sur les fondements et problèmes de l'identification des minzu, Fei Xiaotong décrivait déjà la situation des Baima, également appelés "Tibétains de Pingwu », tout comme il traitait des cas particuliers des Deng (nonofficiellement reconnus) et des Sherpa de la région autonome du Tibet, des Jiarong (rGyal rong) des préfectures de Aba (rNga ba) et Ganzi (dKar mdzes) au Sichuan, ou encore des Premi tibétanisés (reconnus comme Zang au Sichuan, et Pumi au Yunnan $)^{78}$. Autant de cas problématiques d'identités hybrides, ou de revendications de reclassification qui, dans le cas des groupes «tibétains », c'est-à-dire assimilés à la catégorie Zang, représentent parfois aux yeux de certains une tentative de fragmentation et une atteinte à l'intégrité de l'identité tibétaine.

Plusieurs des cas évoqués jusqu'ici illustraient ces tensions liées au processus d'identification. Tenzin Jinba évoque dans le cas des Jiarong, comment, suite à des pressions de l'élite, la reconnaissance d'une «nationalité » Jiarong fut abandonnée au profit de l'inclusion à la catégorie Zang ${ }^{79}$. Les Deng du sud-est du Tibet présentent un cas similaire et bien qu'ayant demandé une identification séparée, ils se sont vu opposer le risque d'une division des tibétains et de leur territoire. De possibles scissions des « branches » de la nationalité Yi peuvent pareillement susciter

77. Yunnan sheng bianwei hui 云南省编委会, éd., Nuzu shebui lishi diaocha 怒族社会历史调查 (Enquêtes sur l'histoire et la société des Nu), Kunming, Yunnan renmin chubanshe, 198I, p. 3. Un groupe appelé Zauzou (Ruoruo 若若 ou Ruorou 若柔 en chinois) se trouvant dans la souspréfecture de Lanping y est maintenant officiellement adjoint. Comme bien d'autres nationalités, sont donc reconnues des «branches》(fenxi 分系 ou zhixi 支系), distinctions sans valeur dans les classifications officielles mais qui viennent dans l'usage commun souligner l'existence de souscatégories (linguistiques ou culturelles). Pour ce qui est des Dulong, j’ai eu l'occasion de montrer que l'accession au statut de minzu est généralement présentée comme la simple concrétisation de la volonté des Drung eux-mêmes ; voir S. Gros, "The Politics of Names: The Identification of the Dulong of Northwest Yunnan," China Information, vol. I8, n 2, 2004, p. 275-302.

78. Fei Xiaotong, "Guanyu woguo minzu de shibie wenti ».

79. Tenzin Jinba, In the Land of the Eastern Queendom: The Politics of Gender and Ethnicity on the Sino-Tibetan Border, Seattle, University of Washington Press, 20I4, p. 2I-34. 
des tensions et être des questions politiquement sensibles, qui ne sont donc pas propres au contexte tibétain ${ }^{80}$.

Le cas des Baima fut l'objet d'une attention particulière alors que dans les années 1970 la question de leur identification comme minzu distinct fut soulevée. Le nom de Baima, évoqué précédemment, renvoie à l'une des principales tribus Di mentionnée par l'historien Sima Qian (I45-90 av. J.-C.). C'est à ce nom et à cette lointaine ancestralité que, depuis les années 1970, les Baima d'aujourd'hui se rattachent. Plusieurs équipes de recherche de diverses institutions menèrent des enquêtes et aboutirent à la conclusion de leur différence d'avec les Tibétains. De manière assez paradoxale, si aujourd'hui le lien entre les Baima et les Tibétains semble être largement reconnu, leur « descendance » des anciens Di a en même temps acquis un statut de fait en Chine, si bien qu'outre leur inclusion soit à l'ensemble Zang, soit à l'ensemble Qiang, il fut également question de les rattacher à un anachronique ensemble Di (Dizu 氏族) ${ }^{8 \mathrm{I}}$. Ils restèrent néanmoins identifiés comme Zang (Tibétains), et selon S. Harrell des rumeurs circulant dans le milieu académique évoquaient le rôle du $\mathrm{Io}^{\mathrm{e}}$ Panchen Lama qui se serait opposé à la séparation des Baima d'avec les Zang. Cette opposition existait ailleurs, et Janet Upton s'est fait l'écho des débats plus académiques et de la perspective historiographique proprement tibétaine à travers le travail de l'historien Muge Samten (dMu dge bSam gtan), qui a ouvertement défendu une identité tibétaine inclusive des groupes tels que les Baima (Dwags po) ${ }^{82}$. Cet auteur arguait notamment que les Baima étaient en fait des descendants d'armées tibétaines envoyées dans ces régions frontières entre le VIII $^{\mathrm{e}}$ et le $\mathrm{IX}^{\mathrm{e}}$ siècle ; d'où leur nom de Baima, dérivé de Bod dmag, « soldat tibétain ». Il évoquait pareillement la manipulation politique du cas des Pumi, dont le nom ne serait autre que Bod mi, «Tibétain $»^{83}$.

Parmi ces « sous-groupes » de nationalité Zang figurent les Ersu, classifiés comme Tibétains en 1964 mais qui, jusqu'au recensement de I990, continuaient pour certains à s'identifier comme Fanzu : la dénomination Fan était alors encore employée sur les états-civils (bukou 户口) des Ersu du district de Ganluo ${ }^{84}$. Wu Da a mis en évidence que dès 1954 les Ersu de Ganluo avaient soumis une demande de reconnaissance à l'occasion de la première session du Congrès du peuple de la Province du Xikang, peu après effacée de la carte et intégrée à la province du Sichuan. La même demande

80. Harrell, Ways of Being Ethnic..., p. 4I, 177, 210.

8I. Voir par exemple Huang Guangxue et Shi Lianzhu, Zhongguo de minzu sbibie, p. 237-242.

82. Janet L. Upton, "Notes Toward a Native Tibetan Ethnology: An Introduction to and Annotated Translation of dMu dge bSam gtan's Essays on Dwags po (Baima Zangzu)," The Tibet Journal, vol. 25, n⿳亠口冋, 2000, p. 3-26.

83. Ces reconstructions sont cependant mal étayées d'un point de vue linguistique ; voir par exemple la critique dans K. Chirkova, 《Baima Zangzu wei Dizu shuo zhiyi » 白馬藏族為氏族說質疑 (The Baima Tibetans and the Di people of Chinese historical records: Challenging the link), Bulletin of Chinese linguistics 中國語言學集刊, vol. 3, nº I, 2008, p. I67-I80.

84. Wu Da 巫達, «Lixing xuanze yu zuqun neixin qinggan: Zhongguo Sichuan Ersuren zuqun rentong de ge'an yanjiu » 理性選擇與族群內心情感——中國四川爾蘇人族群認同的個案研究 (Choix rationnel et primordialisme : une étude de cas sur l'ethnicité des Ersu du Sichuan, Chine), Taiwan renlei xuekan 台灣人類學刊 Taiwan Journal of Anthropology, vol. 4, nº I, 2006, p. I13-147.

(C) École française d'Extrême-Orient, Paris, 20I5

Do not circulate without permission of the editor / Ne pas diffuser sans autorisation de l'éditeur 
fut ainsi renouvelée auprès des autorités du Sichuan, mais tomba dans l'oubli lors de la Révolution culturelle. C'est en 1983 que certains représentants ont de nouveau pétitionné pour demander une reconnaissance en tant que "nationalité » Ersu, ce qui ne s'est pas fait sans l'opposition d'autres Ersu qui se revendiquaient comme tibétains et voyaient les avantages d'une telle affiliation ${ }^{85}$. Pour les deux camps, les motivations apparaissent essentiellement politiques et économiques. Cette opposition quant à l'affiliation au minzu approprié masque cependant un accord de principe concernant leur ethnicité : en effet, qu'ils revendiquent une «nationalité » Zang ou Ersu, ils se disent néanmoins pareillement Ersu, leur autodésignation commune. Wu Da souligne avec justesse la manière dont les sentiments primordiaux à la base de l'identité s'ajustent avec des critères de choix rationnel qui permettent de mettre en lumière la manière dont les identités se construisent en contexte.

\section{Discussion}

L'évolution de la taxinomie en tant que forme de classification des groupes humains, et la diversité des cas de figures évoqués dans cet article, ont permis de souligner la contingence des catégorisations de type ethnique. À la suite de Susan Blum, on pourrait se demander si la question de l'ethnicité en Chine n'est pas l'objet d'une attention démesurée par rapport à ce que le contexte chinois pourrait nous apprendre ${ }^{86}$. Or, les spécificités du cas chinois méritent notre attention précisément parce que le problème de la constitution des groupes ethniques reste sujet à controverse. Nous n'avons pas cessé de nous interroger sur la manière dont les catégories de minzu en viennent à être acceptées et faire partie du sens commun, alors qu'elles nous paraissent tellement arbitraires et politisées. La relation ambiguë entre ce système rigide de classification et les dynamiques de l'ethnicité ajoute à la confusion. C'est cette ambiguïté qu'il convient de prendre pleinement en compte.

Comme le sociologue Ma Rong l'a souligné (avec toutefois une visée politique), le système officiel, via les catégories de minzu, rappelle sans cesse aux personnes qu'elles «appartiennent à un 'groupe spécifique' », et "crée des barrières institutionnelles à l'interaction et l'intégration entre les membres de différents groupes ethniques ${ }^{87}$. Cependant, la structure hégémonique imposée par l'État n'existe pas indépendamment des interactions au niveau local. Il faut ainsi prendre en compte,

85. Pour ces derniers, l'évolution favorable des politiques à l'égard des minorités et notamment des Tibétains à l'époque de Hu Yaobang 胡耀邦 au début des années i980 joua un rôle dans la prise en compte des possibles avantages à faire partie d'une «nationalité » numériquement importante et jouissant d'une reconnaissance internationale. Voir Wu Da, «Lixing xuanze yu zuqun neixin qinggan », p. I29-I3I.

86. Susan D. Blum, "Margins and Centers: A Decade of Publishing on China's Ethnic Minorities," The Journal of Asian Studies, vol. 6I, nº 4, 2002, p. I287-I3IO.

87. Ma Rong, "A New Perspective in Guiding Ethnic Relations in the Twenty-First Century: 'De-Politicization' of Ethnicity in China," Asian Ethnicity, vol. 8, n 3, 2007, p. 2II. 
suivant la proposition de S. Harrell, les différents niveaux de langage relatif à l'ethnicité : le triangle des discours allant du discours étatique de l'identification ethnique au discours académique de l'ethnohistoire, en passant par le discours ordinaire à l'échelle locale ${ }^{88}$.

J'ai dans cet article tenté de mettre en lumière, à propos de la région des confins sino-tibétains, comment ces différents niveaux de langages peuvent être mobilisés, et l'importance de replacer la variabilité des formes de classification (taxinomie) et d'attribution catégorielle (ethnonymie) dans le temps. La manière dont les catégories qui nous sont aujourd'hui connues se sont cristallisées est directement liée à une histoire qui ne débute pas nécessairement dans les années 1950 avec le projet d'identification des minzu, mais s'inscrit dans une histoire plus longue des interactions entre le centre et sa périphérie, et entre les groupes en présence localement eux-mêmes.

Il serait envisageable de poursuivre ce parcours et de multiplier les études de cas qui viendraient alors probablement confirmer la variabilité contextuelle des assignations et revendications identitaires. Dans bien des cas, certains des groupes considérés sont traités comme des groupes (culturels) locaux, plutôt que des groupes ethniques ou collectivités pouvant accéder au statut de minzu. Si bien que des tendances primordialistes ou essentialistes, rationnelles ou situationnelles, seraient toutes probablement présentes en tant que modalités de l'expression identitaire en fonction des contextes d'interaction et des représentations mobilisées, ou bien comme stratégies adaptatives, différentes "manières d'être ethnique » ${ }^{89}$, face à l'expansion han ou tibétaine.

Ces tendances sont nécessairement différentes de ce que l'analyse doit être à même de construire sans se faire simplement le relais des spécificités du langage identitaire local. Ce dernier est habituellement aux mains d'une élite qui fonde ses revendications sur un mode souvent essentialiste, et à des fins politiques. Une forme de pensée généalogique est ainsi généralement mobilisée, qui vise à établir l'« aura de filiation » qui accompagne l'affirmation identitaire. Comme j'ai tenté de le souligner, cette pensée généalogique se décline de diverses manières selon les contextes et les échelles, au niveau local et au niveau national.

Au niveau local, la démarche généalogique est mobilisée pour inscrire le groupe dans un parcours historique qui prend la forme d'une ascendance et sur cette base permet de justifier du bien-fondé du sentiment identitaire - congruent ou non avec les catégories officielles. C'est ainsi souvent l'histoire linéaire, plus que la culture ou la langue, qui vient fournir l'idiome principal des revendications, ou discussions, liées à l'ethnicité, et comme il a souvent été relevé, le mythe d'une histoire commune est un référent identitaire d'une très grande généralité. Encore ne faut-il pas négliger l'importance, notamment au niveau des communautés villageoises, de l'inscription territoriale comme facteur déterminant des identités qui, comme souvent dans les régions montagneuses, s'enracinent dans la topographie et le paysage.

88. S. Harrell, Ways of Being Ethnic, p. 34-35.

89. Ibid., p. 320-325. 
Le discours officiel en RPC autour de la notion d'« État multinational» (duominzu guojia) se fonde quant à lui sur une mentalité généalogique qui se déploie et incorpore les identités locales au sein d'histoires supra-locales qui contribuent à former le discours national sur l'origine et le développement historique de la Chine. Cette mentalité produit un amalgame entre culture, nation, race et généalogie, qui assigne aux groupes non-Han une position ambiguë : bien que composante essentielle de la grande famille nationale (minzu da jiating 民族大家庭), leur descendance de groupes non-Han identifiés comme tels depuis l'antiquité les exclut de fait d'une conception purement généalogique de la nation ${ }^{90}$. Les fondements généalogiques de telles formulations ne peuvent donc pas intégrer le fait interethnique comme producteur de catégories hybrides.

Certains auteurs ont justement mis en évidence qu'une approche de l'ethnicité comme forme d'attribution catégorielle en contexte d'interaction devrait alors s'enrichir de la prise en compte de la manière dont ce rapport distinctif à l'autre peut reposer sur un ensemble de significations partagées ${ }^{91}$. Un tel «idiome commun » se

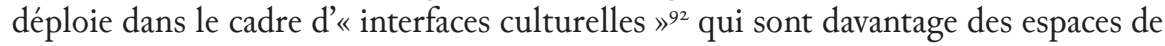
négociations que les lieux d'une acculturation homogénéisante. Grégoire Schlemmer a montré que, dans un contexte géographique et historique similaire, les populations de ce qu'il nomme les «marges sino-indiennes ", sont en interaction entre elles autant qu'avec les centres de pouvoir, et dans ce cadre contribuent de diverses manières à produire « à la fois de la différence - qui marque leur identité et leur existence en tant qu'entité propre - et de l'unité - qui les font se penser comme participant d'un

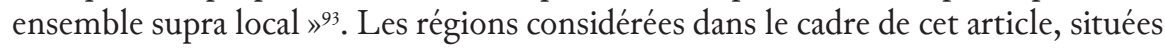
à la rencontre des sphères d'influences chinoise et tibétaine, sont comme nous l'avons vu un exemple assez révélateur de ce type d'interfaces où échanges et interactions ont contribué à l'émergence de similitudes comme de spécificités sociales, culturelles ou religieuses. La production des taxinomies et classifications et leur intrusion dans la vie locale, le triangle des discours sur l'ethnicité évoqué plus haut, participent de la reproduction des structures conceptuelles et institutionnelles.

Les processus de différentiation ou de rapprochement qui en découlent font de cette région des confins sino-tibétains une zone propice au renouvellement d'une réflexion sur l'ethnicité dans le contexte chinois dont cet article ne fait que poser les prémisses. Avant de pouvoir alimenter une telle réflexion à partir d'études de

90. Ambiguïté notamment relevée par Frank Pieke dans un autre contexte. Cf. F. Pieke, "The Genealogical Mentality in Modern China," The Journal of Asian Studies, vol. 62, n I, 2003, p. IOI-I28. Pour une version représentative de la manière dont le discours savant sur le développement historique de l'unité de la Chine intègre la diversité interne, voir Xu Jieshun, "Understanding the Snowball Theory of the Han Nationality," dans T. Mullaney, J. Liebold, S. Gros, E. vanden Bussche, éds., Critical Han Studies. The History, Representation, and Identity of China's Majority, Berkeley, University of California Press, 2012, p. II3-I27.

91. Voir B. Brac de la Perrière, B. Formoso et M. Picard, «L'ethnicité reconsidérée : éclairages sud-est asiatiques ", Aséanie, n 2I, 2008, p. I3I-I46.

92. Ibid., p. $\mathrm{I} 35, \mathrm{I} 38$

93. G. Schlemmer, «Rituels, territoires et pouvoirs dans les marges sino-indiennes », Moussons, $\mathrm{n}^{\circ} \mathrm{I} 19,2 \mathrm{I} 2$, p. 5-18.

(C) École française d'Extrême-Orient, Paris, 20I5 Do not circulate without permission of the editor / Ne pas diffuser sans autorisation de l'éditeur 
cas complémentaires à celles évoquées au cours de cet article, j’ai ici simplement cherché à montrer la nécessité d'une approche régionale visant à prendre en compte les interactions entre plusieurs groupes dans des contextes spécifiques, et à des échelles différentes. En soulignant la labilité des classifications ethniques ainsi que les changements d'identité, et les possibles disjonctions entre les catégories assignées, la culture, la langue et l'identité telle qu'elle est perçue localement, j'ai posé quelques jalons qui peuvent contribuer à affiner notre perception d'une catégorie telle que celle des Zang (Tibétains), et aider à penser certaines formes d'interethnicité dans le contexte chinois.

Keywords: Sino-Tibetan borderlands, Ethnic categorization, Ethnic change, Ethnogenesis, Tibeto-burman. 


\section{Bibliographie}

\section{Amselle, Jean-Loup}

1990 Logiques métisses. Anthropologie de l'identité en Afrique et ailleurs, Paris, Payot.

Bazin, Louis et Hamilton, James Russel

1991 «L'origine du nom Tibet », dans Ernst Steinkellner, éd., Tibetan History and Language: Studies Dedicated to Uray Géza on His Seventieth Birthday, Vienne, Arbeitskreis für Tibetische und Buddhistische Studien, Universität Wien, p. II-I7.

Blum, Susan D.

2002 "Margins and Centers: A Decade of Publishing on China's Ethnic Minorities," The Journal of Asian Studies, vol. 6I, n 4, p. I287-I310.

Brac de la Perrière, Bénédicte, Formoso, Bernard et Picard, Michel

2008 «L'ethnicité reconsidérée : éclairages sud-est asiatiques », Aséanie, n² 2I, p. I3I-I46.

Brown, Melissa J., éd.

1996 Negotiating Ethnicities in China and Taiwan, Berkeley, University of California Press.

Chavannes, Édouard

1913 "Documents historiques et géographiques relatifs à Li-Kiang », dans J. Bacot, Les Mo-So, Leiden, E. J. Brill, p. I27-215.

Chirkova, Katia

2007 "Between Tibetan and Chinese: Identity and Language in the Chinese Southwest," South Asia: Journal of South Asian Studies, vol. 30, n 3 , p. $405-417$.

2008 《Baima Zangzu wei Dizu shuo zhiyi » 白馬藏族為氏族說質疑 (The Baima Tibetans and the Di people of Chinese historical records: Challenging the link), Bulletin of Chinese linguistics 中國語言學集刊, vol. 3, nº I, p. I67-I80.

DAvies, Henry Rodolph

1909 Yünnan. The Link Between India and the Yangtze, Cambridge, Cambridge University Press.

DikÖTTER, Frank

1992 The Discourse of Race in Modern China, Stanford, Stanford University Press. 
Drocourt, Zhitang

2010 «Des simples sauvages aux redoutables étrangers : la notion de « barbares » en Chine ancienne à travers leurs dénominations », dans Isabelle Rabut, éd., Visions du «barbare » en Chine, en Corée et au Japon, Actes de la journée d'étude organisée le 3r mars 2008 par le Centre d'Études Chinoises et le Centre d'Études Japonaises de l'INALCO, Paris, INALCO Éditions, p. $13-28$.

FAN Chuo 梵绰

1961 Man sbu 蠻書, traduit en anglais par G. H. Luce sous le titre Man sbu, Book of the Southern Barbarians, [G.P. Oey, éd.] Southeast Asia Program, Department of Far Eastern Studies, Cornell University, Ithaca, New York.

FEI Xiaotong 费孝通

1980 《Guanyu woguo minzu de shibie wenti » 關於我國民族的識別問題 (Ethnic Identification in China), Zhongguo shebui kexue 中國社會科學 (Social Science in China), $\mathrm{n}^{\circ}$ I, p. I47-162.

Fiskesjö, Magnus

1999 "On the "raw" and the "cooked" barbarians of imperial China," Inner Asia, nos. I-2, p. I39-168.

2006 "Rescuing the Empire: Chinese Nation-Building in the Twentieth Century," European Journal of East Asian Studies, vol. 5, nº 2, p. 15-44.

2011 "The Animal Other: China's Barbarians and their Renaming in the Twientieth Century," Social Text, vol.29, nº 4, p. 57-79.

GELEI 格勒

2008 Zangxue, renleixue lunwen ji 藏學、人類學論文集 (Recueil d'écrits en tibétologie et anthropologie), vol. I, Beijing, Zhongguo zangxue chubanshe.

Gros, Stéphane

2004 "The Politics of Names: The Identification of the Dulong of Northwest Yunnan," China Information, vol. I8, nº 2, p. 275-302.

2009 «Le lieu du pouvoir. Mémoire et enjeu de l'histoire chez les Drung du Yunnan (Chine) ", dans Gisèle Krauskopff, éd., Les faiseurs d'bistoires, Nanterre, Société d'ethnologie, p. 187-215.

Guldin, Gregory E.

1993 The Saga of Anthropology in China: From Malinowski to Moscow to Mao, London, M. E. Sharpe.

2008 State and Ethnicity in China's Southwest, Leiden, Brill.

Gyilung, Tashi Gyatso et Gyilung, Thugchock Dorji

2009 The Treasure of the Ancestral Clans of Tibet, Yeshi Dhondup (trad.), Dharamsala, Library of Tibetan Works and Archives.

HaRRell, Stevan et Li Yongxiang

2003 "The History of the History of the Yi, Part II," Modern China, vol. 29, $\mathrm{n}^{\circ} 3$, p. 362-96. 
HARRELL, Stevan

1990 "Ethnicity, Local Interests, and the State: Yi Communities in Southwest China," Comparative Studies in Society and History, vol. 32, n ${ }^{\circ} 3$, p. 515548.

1995a "Introduction: Civilizing Projects and Reaction to Them," dans S. Harrell, éd., Cultural Encounters on China's Ethnic Frontiers, Seattle, University of Washington Press, p. 3-36.

1995b "The History of the History of the Yi," dans S. Harrell, éd., Cultural Encounters on China's Ethnic Frontiers, Seattle, University of Washington Press, p. 63-9I.

1996 "The Nationalities Question and the Prmi Prblem [sic]," dans M. J. Brown, éd, Negotiating Ethnicities in China and Taiwan, Berkeley, University of California Press, p. 274-296.

2001 Ways of Being Ethnic in Southwest China, Seattle, University of Washington Press.

HaRrell, Stevan, éd.

1995 Cultural Encounters on China's Ethnic Frontiers, Seattle, University of Washington Press.

Hon, Tze-Ki

1996 "Ethnic and Cultural Pluralism: Gu Jiegang's Vision of a New China in His Studies of Ancient History," Modern China, vol. 22, n 3, p. 315-339.

HuANG Guangxue 黄光学 et SHI Lianzhu 施联朱

2005 Zhongguo de minzu sbibie: 56 ge minzu de laili 中國的民族識別— 56 各民族 的來歷 (L'identification ethnique en Chine. L'origine des 56 nationalités), Beijing, Minzu chubanshe.

Huber, Toni, éd.

1999 Sacred Spaces and Powerful Places in Tibetan Culture. A Collection of Essays, Dharamsala, Library of Tibetan Works and Archives.

JaCQues, Guillaume et Michaud, Alexis

2011 "Approaching the Historical Phonology of Three Highly Eroded SinoTibetan Languages: Naxi, Na and Laze," Diachronica, vol. $28, \mathrm{n}^{\circ} 4$, p. $468-498$.

JAGOU, Fabienne

2006 «Vers une nouvelle définition de la frontière sino-tibétaine : la Conférence de Simla (1913-19I4) et le projet de création de la province de Xikang », Extrême-Orient, Extrême-Occident, nº 28, p. I47-167.

Kaup, Katherine P.

2000 Creating the Zhuang: Ethnic Politics in China, Boulder, Lynne Rienner Publishers.

KleEman, Terry F.

1998 Great Perfection: Religion and Ethnicity in a Chinese Millennial Kingdom, Honolulu, University of Hawai'i Press.

(C) École française d'Extrême-Orient, Paris, 20I5 Do not circulate without permission of the editor / Ne pas diffuser sans autorisation de l'éditeur 
KLIEGER, P. Christiaan, éd.

2006 Tibetan Borderlands: Proceedings of the Tenth Seminar of the IATS, 2003, volume 2, Leiden, Brill.

Kyong-McClain, Jeff et Jing Geng

2011 "David Crockett Graham in Chinese Intellectual History: Foreigner as Nation Builder," dans D. M. Glover, S. Harrell, C. F. McKhann, et M. B. Swain, eds., Explorers and Scientists in China's Borderlands I880-1950, Seattle, University of Washington Press, p. 2II-239.

LAPolla, Randy J.

2003 “Overview of Sino-Tibetan Morphosyntax," dans G. Thurgood, and R. J. LaPolla, eds., The Sino-Tibetan Languages, London, Routledge, p. 22-42.

LEIBOLD, James

2007 Reconfiguring Chinese Nationalism: How the Qing Frontier and its Indigenes Became Chinese, New York, Palgrave Macmillan.

Li Shaoming 李紹明

2007 《Zhongguo renleixue de Huaxi xuepai»中國人類學的華西學派, Zhongguo renleixue pinglun 中國人類學評論, $\mathrm{n}^{\circ} 4$, p. 41-63.

LiN Yaohua 林耀华

1987 《Zhongguo Xinan diqu de minzu shibie »中國西南地區的民族識別 (L'identification des minzu dans le Sud-Ouest de la Chine), dans Yunnansheng Bianjizu 云南省编辑组, éd., Yunnan shaoshu minzu shebui lishi diaocha ziliao buibian 雲南少數民族社會歷史調查資料彙編, vol. III, Kunming, Yunnan minzu chubanshe, p. I-6.

Liščák, Vladimir

1993 "The Origins of Ethnology in China: A Survey (From the Mid-I89os Till the Mid-r940s)," Archív Orientální, nº 6I, p. 4I-62.

MA Changshou 馬長壽

2003 [1936] 《Zhongguo Xi'nan minzu fenlei » 中國西南民族分類 (Classification des groupes ethniques du sud-ouest de la Chine), dans Ma Changshou, Ma Changshou minzu xue lunwen ji 馬長壽民族學論文集, Beijing, Renmin chubanshe, p. 49-82.

MA Jianxiong

2009 "Local Knowledge Constructed by the State. Reinterpreting Myths and Imagining the Migration History of the Lahu in Yunnan, Southwest China," Asian Etbnology, vol. 68, n I, p. III-I29.

MA Rong

2007 "A New Perspective in Guiding Ethnic Relations in the Twenty-First Century: 'De-Politicization' of Ethnicity in China,' Asian Ethnicity, vol. 8, $\mathrm{n}^{\circ}$ 3, p. 197-217. 
Mathieu, Christine

2003 History and Anthropological Study of the Ancient Kingdoms of the Sino-Tibetan Borderland: Naxi and Mosuo, Lewiston, The Edwin Mellen Press.

McKhann, Charles F.

2003 "Sacred trails: Genealogical Mapping and the Creation of Historical Space Among the Naxi of Southwest China," Histoire et Anthropologies, Asies 2, p. 29-47.

Mullaney, Thomas

2004 "Ethnic Classification Writ Large: The 1954 Yunnan Province Ethnic Classification Project and Its Foundations in Republican-Era Taxonomic Thought," China Information, vol. I8, nº 2, p. 207-24I.

2011 Coming to Terms with the Nation: Ethnic Classification in Modern China, Berkeley, University of California Press.

Pelliot, Paul

1904 "Deux itinéraires de Chine en Inde à la fin du viII siècle », Bulletin de l'École française d'Extrême-Orient, vol. 4, nos. I-3, p. I3I-413.

PIEKe, Frank N.

2003 "The Genealogical Mentality in Modern China," The Journal of Asian Studies, vol. 62, n I, p. IOI-I28.

Pommaret, Françoise

1999 "The Mon-pa revisited: In search of Mon," dans Toni Huber, éd., Sacred Spaces and Powerful Places in Tibetan Culture. A Collection of Essays, p. 52-73.

REN Naiqiang 任乃强

2009 Xikang tujing 西康圖經, dans Ren Naiqiang, Ren Naiqiang Zangxue wenji 任乃强藏學集, vol I, Beijing, Zhongguo zangxue chubanshe, p. 205-210.

Roche, Gerald

2014 "The Vitality of Tibet's Minority Languages in the Twenty-First Century," Multiethnica, ${ }^{\circ} 35$, p. 24-33.

SCHLEMMER, Grégoire

2012 «Rituels, territoires et pouvoirs dans les marges sino-indiennes », Moussons, $\mathrm{n}^{\circ}$ 19, p. 5-18.

SHELACH, Gideon

1996 "The Qiang and the Question of Human Sacrifice in the Late Shang Period," Asian Perspectives, vol. 35, n' I, p. I-26.

SHI Shuo 石硕

2008 «Zang-Yi zoulang diqu Zang-Mian yu minzu qiyuan wenti yanjiu pingshu » 藏彝走廊地區藏緬語民族起源問題研究評述, Xixiang zbanxian 思想戰線, vol. 34, nº 2, p. 15-19.

(C) École française d'Extrême-Orient, Paris, 2015 Do not circulate without permission of the editor / Ne pas diffuser sans autorisation de l'éditeur 
SHIH Chuan-kang

2010 Quest for Harmony: The Moso Traditions of Sexual Union and Family Life, Stanford, Stanford University Press.

SHNEIDERMAN, Sara

2006 "Barbarians at the Border and Civilising Projects: Analysing Ethnic and National Identities in the Tibetan Context," dans P. C. Klieger, éd., Tibetan Borderlands, Proceedings of the Tenth Seminar of the IATS, 2003, volume 2, Leiden, Brill, p. 9-34.

Stein, Rolf A.

1956 «Les K’iang des marches sino-tibétaines, exemple de continuité de la tradition », dans École pratique des hautes études, Section des sciences religieuses, Annuaire 1957-1958, Paris, Imprimerie nationale, p. 3-15.

1959 Les Tribus anciennes des marches sino-tibétaines, Université de Paris, Faculté des Lettres, Imprimerie nationale.

1987 La Civilisation tibétaine, Paris, L’Asiathèque.

Sтотт, Wilfrid

1963 "The expansion of Nan-chao Kingdom: Between the Years A.D. 750-86o and the Causes That Lay Behind It as Shawn in the T'ai-ho Inscription and the Man Shu," T'oung Pao, vol. L, nos. I-3, p. 190-220.

Sun Hongkai 孙宏开

2009 "Language Recognition and Nationality," International Journal of the Sociology of Language, vol. 97, $\mathrm{n}^{\circ}$ I, p. 9-22.

1990 "Languages of the Ethnic Corridor in Western Sichuan" (R. J. LaPolla, trad.), Linguistic of the Tibeto-Burman Area, vol. I3, n ${ }^{\circ}$ I, p. I-3I.

1983 《Liujiang liuyu de minzu yuyan ji qi xishu fenlei » 六江流域的民族語言 及其係數分類, Minzu xuebao 民族學報, vol. 3, p. 196-213.

SutTon, Donald S.

2007 "Ritual, Cultural Standardization, and Orthopraxy in China: Reconsidering James L. Watson's Ideas," Modern China, vol. 33, n 3, p. 3-2I.

TAO Yunkui 陶云迬

2012 [194I] 《Yunnan tuzhu minzu yanjiu zhi huigu yu qianzhuan » 雲南土著 民族研究之回顧與前瞻 (Rétrospective et perspectives des études sur les ethnies indigènes du Yunnan), dans Tao Yunkui, Tao Yunkui minzu yanjiu wenji 陶云迬民族研究文集, Beijing, Minzu chubanshe, p. 92-IIo.

TENZIN Jinba

2014 In the Land of the Eastern Queendom: The Politics of Gender and Ethnicity on the Sino-Tibetan Border, Seattle, University of Washington Press.

Thoraval, Joël

1990 "Le concept chinois de nation est-il 'obscur' ? À propos du débat sur la notion de minzu dans les années 1980 ", Bulletin de sinologie, vol. 65, p. 24-4I. 
TOURnADRe, Nicolas

2005 «L'aire linguistique tibétaine et ses divers dialectes », Lalies, Paris, Presses de l'ENS, Editions Rue d'Ulm, n' 25, p. 7-56.

Upton, Janet L.

2000 "Notes Toward a Native Tibetan Ethnology: An Introduction to and Annotated Translation of dMu dge bSam gtan's Essays on Dwags po (Baima Zangzu)," The Tibet Journal, vol. 25, n I, p. 3-26.

VAN Driem, George

2013 "The ancestry of Tibetan," dans Gray Tuttle, Kunsang Gya, Karma Dare et Johnathan Wilber, eds., The Third International Conference on Tibetan Language, Volume I: Proceedings of the Panels on Domains of Use and Linguistic Interactions, New York: Trace Foundation, p. 363-397.

WANG Jianmin 王建民 et al.

1998 Zhongguo minzuxue shi 中國民族學史 (Histoire de l'ethnologie chinoise), vol. 2 (1950 1997), Kunming, Yunnan jiaoyu chubanshe.

WANG Mingke 王明珂

2006 [1997] Hua-Xia bianyuan: Lishi jiyi yu zuqun rentong 華夏邊緣——歷史記憶與 族群認同 (Aux frontières de la Chine : Mémoire historique et identité ethnique), Beijing, Shehui kexue wenxian chubanshe.

1999 "From the Qiang Barbarians to the Qiang Nationality The Making of a New Chinese Boundary," dans Shu-min Huang et Cheng-kuang Hsu, eds., Imagining China: Regional Division and National Unity, Taipei, Institute of Ethnology, p. 43-80.

2008 [2003] Qiang zai Han-Zang zbi jian: Chuanxi Qiangzu de lishi renleixue yanjiu 姜在漢藏之間——川西姜族的歷史人類學研究 (Les Qiang entre Han et Tibétains: Une anthropologie historique des Qiang du Sichuan de l'Ouest), Beijing, Zhonghua Shuju.

WELLENS, Koen

1998 "What's in a name? The Premi in Southwest China and the consequences of defining ethnic identity," Nations and Nationalism, vol. 4, n I, p. I7-34.

2010 Religious Revival in the Tibetan Borderlands: The Premi of Southwest China, Seattle, University of Washington Press.

2012 "Migrating Brothers and Party-State Discourses on Ethnic Origin in Southwest China," dans T. Huber et S. Blackburn, eds., Origins and Migrations in the Extended Eastern Himalayas, Leiden, Brill, p. 299-319.

Wu Da 巫達

2004 « Rentong zhi jueze: Sichuan Ersuren zuqun rentong jiangou de minzu zhi yanjiu 》認同之抉擇一一四川爾蘇人族群認同建構的民族志研究, thèse de doctorat, Chinese University of Hong Kong. 
2006 «Lixing xuanze yu zuqun neixin qinggan: Zhongguo Sichuan Ersuren zuqun renting de ge'an yanjiu » 理性選擇與族群內心情感——中國四川爾蘇 人族群認同的個案研究 》(Choix rationnel et primordialisme : Une étude de cas sur l'ethnicité des Ersu du Sichuan, Chine), Taiwan renlei xue kan 台灣人類學刊 Taiwan Journal of Anthropology, vol. 4, nº I, p. II3-I47.

XIANG Da 向达

1962 Manshu jiaozbu 蠻書校注 (Manshu annoté), Beijing, Zhonghua shuju.

Xu Jieshun

2012 "Understanding the Snowball Theory of the Han Nationality," dans T. Mullaney, J. Liebold, S. Gros, E. vanden Bussche, eds., Critical Han Studies: The History, Representation, and Identity of China's Majority, Berkeley, University of California Press, p. II3-I27.

YANG Fuquan 杨福泉

2005 Naxizu yu zangzu lisbi guanxi yanjiu 納西族與藏族歷史關係研究 (Étude des relations historiques entre Naxi et Tibétains), Beijing, Minzu chubanshe.

YANG Shao-yun

2014 "Fan and Han: The Origins and Uses of a Conceptual Dichotomy in Mid-Imperial China, ca. 500-I200,” dans Francesca Fiaschetti et Julia Schneider, eds., Political Strategies of Identity-building in Non-Han Empires in China, Wiesbaden: Harrasowitz Verlag, p. 9-36.

You Zhong 尤中

1994 Yunnan minzushi 雲南民族史, Kunming, Yunnan daxue chubanshe.

YANG Chengzhi 杨成志

2004 [1932] 《Xi'nan minzu de yanjiu » 西南民族的研究 (Étude sur les groupes ethniques du sud-ouest de la Chine), dans Liu Shaorui 刘邵瑞, éd., Yang Chengzhi wenji, Guangzhou 杨成志文集, Zhongshan daxue chubanshe.

YUDRU Tsomu

2013 "Taming the Khampas: The Republican Construction of Eastern Tibet," Modern China, vol. 39, $\mathrm{n}^{\circ}$ 3, p. 319-344.

Yunnan sheng bianji zu 云南省編輯組, éd.

1987 Yunnan shaoshu minzu shebui lishi diaocha ziliao buibian 云南少数民族社會 歷史調查資料彙編 (Compilation de matériaux d'enquêtes sur l'histoire et la société des nationalités minoritaires du Yunnan), vol. III, Kunming, Yunnan minzu chubanshe.

Yunnan sheng bianwei hui 云南省编委会, éd.

1981 Nuzu shebui lishi diaocha 怒族社會歷史調查 (Enquêtes sur l'histoire et la société des $\mathrm{Nu}$ ), Kunming, Yunnan renmin chubanshe.

ZHAO Xinyu 赵心愚

2004 Naxizu yu Zangzu guanxi shi 納西族与藏族關係史 (Histoire des relations entre Naxi et Tibétains), Chengdu, Sichuan renmin chubanshe. 